\title{
Ríos y ciudades: análisis de los usos del suelo en el espacio fluvial de las áreas urbanas españolas
}

Rivers and cities: analysis of land use in the fluvial space of Spanish urban areas

\author{
Francisco Durán Vian \\ fduran@alumni.unav.es \\ Juan José Pons Izquierdo \\ jpons@unav.es \\ Departamento de Historia, Historia del Arte, Geografía y Ordenación del Territorio \\ Universidad de Navarra (España)
}

\section{Resumen}

En el presente artículo se analizan los usos del suelo existentes en las zonas de inundación frecuente de las áreas urbanas españolas. Estos se clasifican en grupos, distinguiendo las coberturas naturales de las antrópicas y, dentro de ellas, los usos compatibles y no compatibles con las inundaciones fluviales. El análisis realizado determina y compara el reparto de usos en el espacio fluvial y en las áreas urbanas, estudiando la ocupación de los ríos según la región biogeográfica a la que pertenecen y su temporalidad de flujo. Los resultados muestran que (1) los ríos en las ciudades son potenciales infraestructuras verdes donde abundan las coberturas naturales, espacios ajardinados, agrícolas y ganaderos; (2) se ha ocupado casi tres cuartas partes del espacio fluvial en las áreas urbanas; y (3) la presión antrópica es mayor en los ríos de la región mediterránea y, sobre todo, en los cursos de caudal intermitente. Este trabajo, pone de 
manifiesto la falta de una ordenación territorial coherente con los espacios fluviales y puede ser útil para contribuir a una sistematización metodológica que permita ampliar y contrastar el conocimiento de esta línea científica.

Palabras clave: río urbano; territorio fluvial; zonas inundables; usos del suelo; infraestructura verde.

\begin{abstract}
This article analyses the existing land-uses in the areas of frequent flooding in Spanish urban areas. These are classified into groups, distinguishing between natural and anthropogenic coverages and, within these, uses that are compatible and not compatible with flooding. The analysis carried out decides and compares the distribution of uses within the fluvial space and the urban areas, studying the occupation of the rivers according to the biogeographical region to which they belong and its temporal flow. The results show that (1) rivers in the cities are potentially green infrastructures where there are plenty of natural coverings, garden areas, agriculture and livestock farming; (2) it has taken up almost three quarters of the fluvial space in urban areas; and (3) the anthropic pressure is greater in the rivers of the Mediterranean region, especially, in the intermittent flow courses. This article, highlights the lack of a coherent spatial planning with the fluvial spaces which can be useful to contribute to a methodological systematization, allowing to expand and contrast the knowledge of this scientific line.
\end{abstract}

Key words: urban river; fluvial territory, flood zones; land use; green infrastructure.

\title{
1 Introducción
}

\subsection{La ocupación de las zonas inundables}

Históricamente, el ser humano ha modificado la superficie terrestre con la finalidad de obtener productos esenciales para su supervivencia, sin llegar a ocasionar con ello grandes cambios en el sistema natural del planeta. No obstante, en los últimos cincuenta años el ritmo de transformación se ha acelerado (Parveen et al., 2018), impactando en la biosfera y en la geosfera más que en ninguna otra época de la historia (Entwistle et al., 2019). Se estima que en la actualidad más del 50\% de la superficie terrestre ya ha sido modificada (Hooke et al., 2012), poniendo en riesgo gran parte de los ecosistemas del planeta.

Tal y como afirman varios estudios (Olson \& Dinerstein, 1998; Entwistle et al., 2019), los ríos son uno de los ecosistemas más perjudicados por esa transformación y su funcionalidad ecológica se 
encuentra en una situación crítica (Buijse et al., 2002; Lewin, 2013). La causa de este deterioro se debe, sobre todo, a la rápida expansión de los usos agrícolas y urbanos (Tockner \& Standford, 2002; Dewan \& Yamaguchi, 2009; Kutschker et al., 2009; Biron et al., 2014; Amoateng et al., 2018) y al crecimiento de la población mundial (Hooke et al., 2012).

Esto es muy comprensible, puesto que la supervivencia del ser humano siempre ha estado ligada a la presencia de agua (Vázquez-Rodríguez, 2017) y las llanuras de inundación han sido lugares tradicionalmente privilegiados para el uso antrópico (Middleton, 2012). De hecho, los fondos de valle ofrecen grandes ventajas socioeconómicas y se han convertido en los principales ejes de articulación y desarrollo económico del territorio (Tockner \& Standford, 2002; Bravard, 2006; López-García et al., 2007; Baena et al., 2016).

Los primeros asentamientos humanos mantuvieron un prudente respeto hacia el río. No obstante, con el paso del tiempo, el crecimiento urbano se orientó hacia los llanos de inundación y muchas actividades productivas ligadas al sector primario e industrial se fueron aproximando a los cauces (López-García et al., 2007; Lerner \& Holt, 2012; Santos \& Peña-Corvillon, 2014; Knoll et al., 2017; Kondolf \& Pinto, 2017).

La expansión urbana se aceleró a mediados del siglo XX, cuando se ocuparon amplios espacios inundables por fábricas, infraestructuras de transporte, edificaciones residenciales y otras dotaciones (García-Codron, 2004). Además, en esta época se ejecutaron numerosas actuaciones de tipo estructural destinadas a controlar los ríos y reducir sus zonas inundables, tanto en el ámbito rural como en el urbano (Ollero et al., 2009; Berga-Casafont, 2011; Conesa \& Pérez, 2014), permitiendo así un mayor acercamiento de todos esos usos a los cauces (Fernández et al., 2015).

A principios de los años setenta, muchos de los espacios agrícolas e industriales ligados a los cursos de agua urbanos perdieron su utilidad y comenzaron a surgir proyectos de regeneración de riberas (Carré \& Deutsch, 2015). Estas intervenciones, vigentes hoy en día, apuestan por la socialización de los ríos (Santasusagna \& Tort, 2018) mediante la sustitución de los usos abandonados o poco valorados en la actualidad, por otros nuevos, entre los que destacan los espacios verdes, las infraestructuras de ocio y las zonas residenciales (Pellicer, 2005; Lerner \& Holt, 2012; Kondolf \& Pinto, 2017).

Estos proyectos, que han ido transformando los corredores fluviales en espacios públicos donde abundan las superficies verdes, reflejan una mayor sensibilización ambiental y comprensión de los sistemas fluviales. Sin embargo, muchos ríos urbanos en España están marcados por una 
etapa de crecimiento urbanístico desmesurado en la que hubo una tendencia muy generalizada a la conquista del espacio fluvial (Pellicer, 2005; González et al., 2007). Se trata de la "década prodigiosa" del urbanismo español, comprendida entre los años 1997 y 2006 (Burriel, 2008).

En este escenario, el crecimiento demográfico y del sector turístico vacacional hizo que aumentase sustancialmente la densidad de viviendas construidas. La fachada mediterránea y Madrid fueron los grandes focos de construcción residencial (Olcina, 2004). Esta avanzó, en parte, a través de terrenos con riesgos de inundación (Asociación Española de Geografía \& Colegio de Geógrafos, 2006).

Por tanto, no es de extrañar que, según se indica en numerosos trabajos (Mateu, 1990; Mateu \& Camarasa, 2000; Arranz-Lozano, 2008; Conesa \& Pérez, 2014; Noguera et al., 2018; Observatorio de la Sostenibilidad, 2019), los ríos de la España mediterránea hayan sido muy afectados por la urbanización, especialmente los ubicados en la zona litoral y las desembocaduras fluviales.

Por otro lado, varios estudios (Olcina, 2004; Doménech et al., 2008; Noguera et al., 2018; Kondolf et al., 2013) señalan la alta ocupación producida en los lechos de pequeños cursos intermitentes: barrancos, ramblas, rieras y arroyos, habiéndose incorporado en algunos casos a la trama urbana. Ejemplo paradigmático son las ramblas, cuyo concepto de origen hidráulico ha adquirido en muchas ciudades mediterráneas una significación urbanística (Pellicer, 2005). Además, este tipo de ríos ha soportado durante miles de años un fuerte aprovechamiento agrícola y aunque la vocación del territorio ha cambiado en las últimas décadas, estos usos aún están muy presentes (Camarasa et al., 2018).

\subsection{Consecuencias de la alteración de la zona inundable}

La ocupación del espacio inundable, la alteración de los cursos de agua y otras acciones humanas sobre las cuencas son responsables de grandes cambios en el paisaje fluvial, así como en sus características geomorfológicas y dinámica hidrológica (Baena, 2006; López-García et al., 2007; Durán et al., 2013). Se estima que esas acciones han causado un enorme deterioro del patrimonio natural del planeta (Tockner \& Standford, 2002; Vietz et al., 2014).

A escala local, el desarrollo de usos vinculados al tejido urbano próximo a los ríos genera numerosos impactos sobre el ecosistema fluvial: alteraciones geomorfológicas en el cauce, eliminación del bosque de ribera, impermeabilización del terreno, aumento de la escorrentía, deterioro de la calidad del agua y limitación de la capacidad de la zona inundable para atenuar 
las avenidas fluviales (Kutschker et al., 2009; Conesa \& Pérez, 2014; Covarrubia et al., 2016; Kellner \& Hubbart, 2016; Martínez et al., 2017).

Por otro lado, la alteración de los llanos de inundación también tiene repercusiones sociales y económicas, dado que, al haber un acercamiento a los cauces, los asentamientos humanos están más expuestos al riesgo de inundación (Mateu, 1990; Adikari \& Yoshitani, 2009; Olcina \& DíezHerrero, 2017; Ollero \& Briz, 2018; Barriendos et al., 2019).

De hecho, en Europa, las inundaciones son el desastre natural más común (Berga-Casafont, 2011) y el que mayor impacto económico tiene (Akter et al., 2018), sobre todo en las áreas urbanas y periurbanas de rápido desarrollo (Nunes et al., 1999).

Desde finales del siglo XIX ha habido un notable incremento de la población afectada por episodios de inundación (Barriendos et al., 2019) y los daños causados por éstos han aumentado más del doble desde mediados del siglo XX (Munich Re Group, 2006; BergaCasafont, 2011). Esto es lógico, pues en la última centuria se implantaron en territorios de riesgo muchos usos no compatibles con la dinámica fluvial (IGME, 2004; Olcina, 2004).

Este panorama es todavía peor en España, ya que ocupa uno de los primeros puestos entre los estados europeos en relación al número de inundaciones anuales y al de víctimas mortales por año asociadas a las avenidas (Berga-Casafont, 2011). Según el Consorcio de Compensación de Seguros y la Dirección General de Protección Civil y Emergencias, cada año se registran una media de 10 episodios de inundación graves (MITECO, 2019). Es el fenómeno natural de mayor impacto en la memoria histórica de los españoles (Núñez et al., 2019) y el que más daños económicos ha provocado (Gallegos-Reina, 2017).

Muchos de estos desastres (incluyendo los de dimensiones catastróficas) se localizaron en ámbitos urbanos, sobre todo litorales, por los que transcurren cursos de agua mediterráneos, cortos y de naturaleza efímera (Ayala-Carcedo, 2002). De hecho, en la segunda mitad del siglo XX las consecuencias más severas están asociadas a inundaciones relámpago o flash floods producidas en esos escenarios (García-Codron, 2004).

\subsection{El concepto de espacio fluvial}

El modelo global de desarrollo urbano responde a una política de ordenación territorial que no ha considerado adecuadamente el espacio inundable (Herrera, 2014). Esto se debe a que, hasta fechas recientes, los cursos de agua se entendían como elementos lineales y no como territorios (Biron et al., 2014; Hernández, 2018). 
Sin embargo, los ríos son ecosistemas cuya extensión va más allá de los propios cauces (Baptist et al., 2004; Piégay et al., 2005; Ollero et al., 2009; Kline \& Cahoon, 2010; Ballais et al., 2011). Las zonas inundables son espacios construidos por la dinámica del agua y están en continuo proceso de remodelación (Ollero et al., 2009). Forman parte integrante del sistema fluvial (EEA, 2018) y absorben el exceso de caudal y sedimentos, desempeñando un papel ecológico esencial (Camarasa \& Bescós, 2004). Estos sistemas, en su conjunto, representan algunos de los paisajes más dinámicos y ricos del planeta (Radcliffe, 1977; Entwistle et al., 2019).

En los últimos veinte años han surgido, desde el ámbito académico, diferentes conceptos basados en criterios hidrogeomorfológicos que vienen a reconocer el espacio funcional de los ríos. Destacan los términos "Streamway", "Inner river zone guideline" y "Channel migration zone" en el ámbito anglosajón, "Espace d'liberte" en Francia y Canadá, "Revitalization space" en Suiza, "Room for the Rivers" en Países Bajos, "Fascia di mobilitá" en Italia y "Territorio fluvial" en España (Ollero et al., 2009; Hernández, 2018).

Aunque cada uno de estos términos tiene una definición particular, los criterios para su delimitación se centran siempre en la movilidad del cauce y en los problemas de inundabilidad (Biron et al., 2014). Todos ellos consideran como territorio del río el cauce, el corredor ribereño y, total o parcialmente, la llanura de inundación (Ollero et al., 2009).

El presente artículo hace referencia a estos términos, de manera genérica, bajo el concepto "espacio fluvial" y, tal y como se explica en el apartado metodológico, se representa con la zona inundable asociada al período de retorno de diez años, también conocida como "zona de inundación frecuente".

\subsection{Factores que configuran el espacio fluvial}

El espacio fluvial es construido por el propio río a lo largo de su historia. Está determinado por factores naturales tales como la climatología, la geología y la pendiente (Ollero et al., 2009; Kondolf et al., 2013). Sin embargo, tal y como se ha señalado en el apartado 1.2., las zonas inundables en los ámbitos urbanos han sido modificadas, por lo que los factores antrópicos también tienen un papel destacado en su configuración.

Considerando los factores naturales, el ámbito climático juega un papel muy relevante en el funcionamiento hidrológico de los cursos de agua y en la delimitación del espacio fluvial (Thornes \& Wainwright, 2004; Ollero et al., 2009; Baena et al., 2016). 
En Europa, los ríos presentan diferentes características hidrológicas. En las regiones de clima continental y oceánico (norte y centro) son generalmente largos y regulares. Sin embargo, en la región mediterránea suelen ser más cortos, muy irregulares y presentan un marcado estiaje (Mateu, 1990; Noguera et al., 2018). Para el caso de la península ibérica están, además, muy influenciados por el relieve (Olcina, 1988).

España posee una gran diversidad de cursos fluviales. Los dos climas más extendidos son el oceánico (al norte del país) y el mediterráneo (centro, este y sur). Las crecidas fluviales en las cuencas de la zona norte son, por lo general, algo más lentas y previsibles, mientras que en la zona mediterránea son más rápidas e irregulares (García-Codron, 2004; García-Codron et al., 2012; Camarasa, 2016). Esto influye sobre la definición de los espacios inundables, pues son más variados y menos advertidos en las regiones de clima mediterráneo y más reconocibles en la zona oceánica (Malavoi \& Souchon, 1989; Mateu, 1989; EWGDF, 2010).

Respecto a los factores antrópicos, las actuaciones destinadas a modificar la zona inundable de los ríos urbanos han sido frecuentes en toda España; no obstante, como se apunta en el apartado 1.1., las referencias bibliográficas indican que los espacios fluviales mediterráneos y los correspondientes a cursos intermitentes son los que se han visto sometidos a más presión.

Las barreras laterales construidas en estos cursos evitan los desbordamientos puntuales de crecidas ordinarias y limitan el espacio fluvial (González et al., 2007; Barriendos et al., 2019). Además, debido a la necesidad de almacenamiento de agua para regar en época estival y a la intención de controlar las crecidas, los cursos mediterráneos están más represados que los de la zona húmeda (Grantham et al., 2010; Kondolf et al., 2013). Esto altera el régimen de caudales y, por tanto, influye en los límites del espacio fluvial original, que pasa de ser más variable e inadvertido a más estable y perceptible.

En el contexto de la regulación de caudales, es importante remarcar que, a pesar de que se hayan construido grandes presas en muchos ríos mediterráneos (Berga-Casafont, 2003), hay una enorme cantidad de cursos efímeros y cortos que no han sido represados. Así pues, generan grandes avenidas naturales tras episodios intensos de precipitación (Ayala-Carcedo, 2002; Arranz-Lozano, 2008). 


\section{Justificación y objetivos del trabajo}

La gestión del riesgo de inundación es un asunto de interés internacional que está ganando protagonismo debido al aumento de población expuesta a inundaciones y a las proyecciones de cambio climático (Madsen et al., 2014; IPCC, 2013).

Desde la implementación de la Directiva de Inundaciones o Directiva 2007/60/CE, las medidas no estructurales para luchar contra las inundaciones han ido adquiriendo un papel destacado (Olcina \& Diez-Herrero, 2017). Los estados miembros, impulsados por la Comisión Europea, están trabajando en la mejora de la gestión del territorio inundable, con intención de evitar y minimizar los daños que producen las avenidas (Berga-Casafont, 2011). Este objetivo debería ser también prioritario para la ordenación territorial y el planeamiento urbanístico, que han de evitar la implantación de usos no compatibles con las inundaciones y fomentar los que son más resilientes a la dinámica fluvial (Gallegos-Reina, 2017; MITECO, 2019).

En esa línea de trabajo se están produciendo importantes avances en los últimos años, sobre todo derivados de la realización de los Planes de Gestión del Riesgo de Inundación por parte de las administraciones hidráulicas (Olcina \& Díez-Herrero, 2017). Uno de los más relevantes es la elaboración de cartografía de inundabilidad, entre la que destacan los mapas de riesgo (BergaCasafont, 2011). Éstos relacionan los usos del suelo con la peligrosidad natural y permiten conocer el grado de exposición y vulnerabilidad ante un episodio de rango extraordinario.

No obstante, a pesar del progreso en el campo normativo y cartográfico, aún hacen falta estudios técnicos y científicos sobre esta materia, que pueden ser de utilidad tanto para la administración, como para el ámbito académico y divulgativo. Con este trabajo se pretende avanzar en esa línea.

La idea de que se han invadido las zonas inundables está ampliamente extendida entre la comunidad científica. Como ya se ha visto, la ocupación del espacio de los ríos -sobre todo en los ámbitos urbanos- se menciona con frecuencia en la literatura académica. También, hay estudios más específicos que abordan la elevada presión que sufren los ecosistemas fluviales mediterráneos y, principalmente, los de caudal intermitente. Sin embargo, no se han encontrado datos cuantitativos sobre el tipo y grado de ocupación antrópica para el conjunto de espacios fluviales del territorio de todo un país.

Conocer el reparto de los usos y coberturas del suelo en el espacio inundable es fundamental para comprender el impacto que tienen las actividades humanas en los ecosistemas fluviales (Yeh 
\& Li, 1999; Dewan \& Yamaguchi, 2009; Covarrubia et al., 2016) e incentivar políticas de desarrollo económico, social y ambiental coherentes con ellos (Long et al., 2007). Además, con estos datos se puede establecer un escenario de referencia para poder comparar la situación actual con otras futuras y realizar un seguimiento periódico de su evolución.

Por tanto, en el presente trabajo se estudian, a escala nacional, las zonas inundables de origen fluvial con objeto de determinar el reparto de usos del suelo en el espacio fluvial de las áreas urbanas, aportando, además, datos sobre la cantidad de superficie destinada al sector residencial y a los usos no compatibles con las inundaciones.

La ocupación del espacio fluvial se analiza según (1) la región biogeográfica a la que pertenecen los cursos de agua y (2) su temporalidad de flujo. ${ }^{1}$

\section{Metodología}

El procedimiento de trabajo desarrollado consta de dos fases. En la primera se efectúa un tratamiento cartográfico de diversas coberturas territoriales y en la segunda se analiza la información obtenida en las operaciones gráficas anteriores.

En la fase de tratamiento cartográfico, primero se crea una capa que recoge los usos del espacio fluvial urbano, y después se identifican esos usos con la zona biogeográfica y el tipo de curso al que pertenecen.

La cartografía base que se emplea corresponde a tres mapas que muestran los usos del suelo, las áreas urbanas y el espacio fluvial. La cartografía complementaria consta de otros dos mapas que representan las regiones biogeográficas y la red hidrográfica. El esquema general de esta primera fase se representa en la Figura 1.

En la fase analítica, primero se comparan los usos en el espacio fluvial urbano con los usos presentes en el conjunto de áreas urbanas que disponen de uno o más ríos y, posteriormente, se examinan los usos en función del ámbito biogeográfico y la temporalidad de flujo del curso al que están asociados. El primer análisis permite generar un escenario de referencia en el contexto urbano y el segundo conocer cómo varía el uso de las zonas inundables según las características físicas de los ríos.

1 Según la ORDEN ARM/2656/2008, de 10 de septiembre, por la que se aprueba la instrucción de planificación hidrológica, los ríos pueden clasificarse en función de la temporalidad de su flujo. Los regímenes son: permanentes, temporales, intermitentes y efímeros. En el presente trabajo, se distingue entre los "permanentes o de caudal circulante de forma habitual" y "no permanentes o de caudal intermitente". 
El trabajo cartográfico se efectúa con el software ArcMap 10.6 de ESRI y el proceso analítico de los resultados con la aplicación Excel de Microsoft.

En los siguientes apartados se mencionan y detallan las bases de datos empleadas y la metodología seguida en la realización del trabajo.

Figura 1. Esquema general de la fase de tratamiento cartográfico

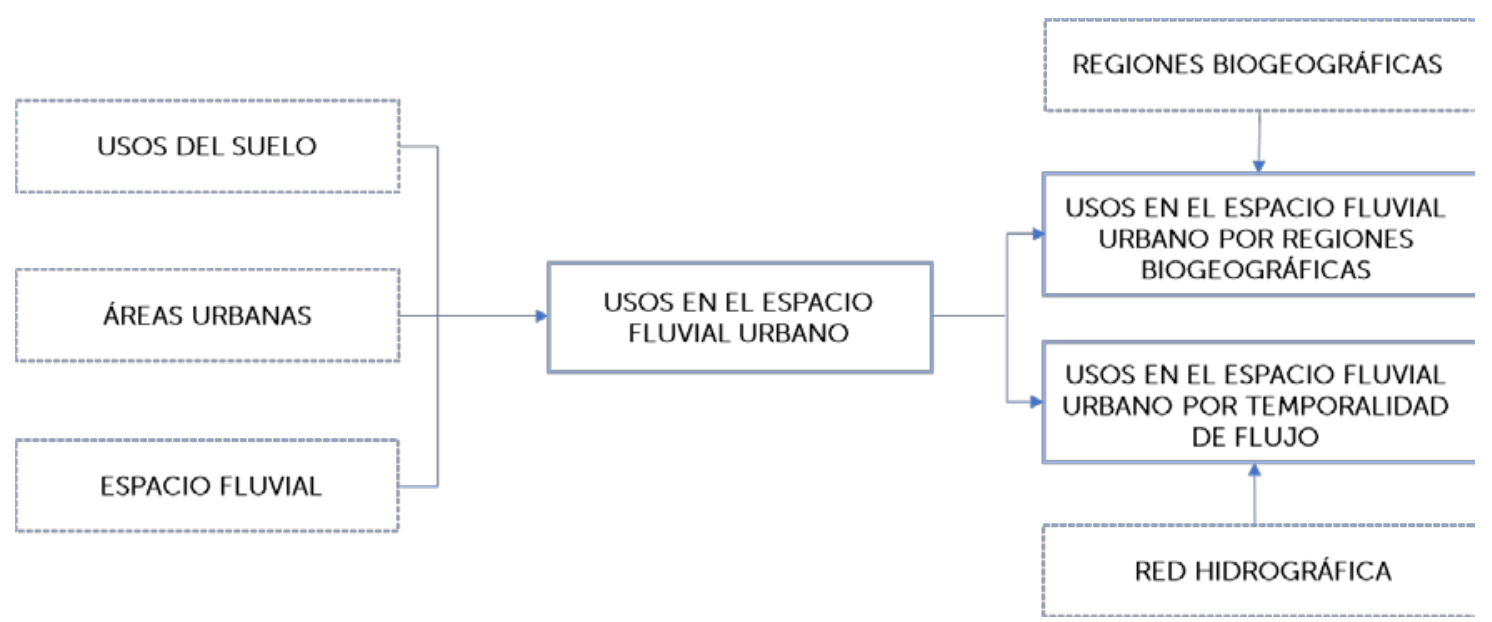

Fuente: elaboración propia

\subsection{Cartografía empleada}

Tras consultar y valorar el uso de diversas fuentes cartográficas, se opta por representar las coberturas de usos de suelo, áreas urbanas y espacio fluvial con los mapas de usos del suelo del Sistema de Información de Ocupación del Suelo de España (SIOSE), las Zonas de Morfología Urbana (ZMU) y las Zonas inundables asociadas al período de retorno de diez años.

Por otro lado, para llevar a cabo el análisis separativo según los ámbitos biogeográficos y la temporalidad de flujo, se utilizan los mapas de regiones biogeográficas de la Agencia Europea de Medio Ambiente (AEMA) y de ríos clasificados según Pfafstetter modificado.

Toda la cartografía se ha obtenido de fuentes oficiales del Gobierno de España y la AEMA. A continuación, se describen los mapas seleccionados.

a) Usos del suelo del SIOSE

Esta base de datos de ocupación del suelo producida por el Instituto Geográfico Nacional se creó en 2005 y se ha actualizado en los años 2009, 2011 y 2014. Se trata de una cartografía realizada para todo el territorio nacional a escala 1:25 000. Es la información gráfica de usos del territorio más detallada que existe para el conjunto de España. 
La base de datos consta de una serie de campos con información de las coberturas presentes en cada polígono. En este trabajo se ha empleado la clasificación de usos del Consejo Directivo de la Infraestructura de Información Geográfica de España (CODIIGE) y de la Directiva europea INSPIRE (HILUCS). Esta doble clasificación de usos permite que la consulta a la tabla pueda hacerse desde perspectivas diferentes. En la Figura 2 se muestra esta cartografía con un agrupamiento de usos que simplifica su representación.

\section{Figura 2. Usos del suelo del SIOSE (representación simplificada)}

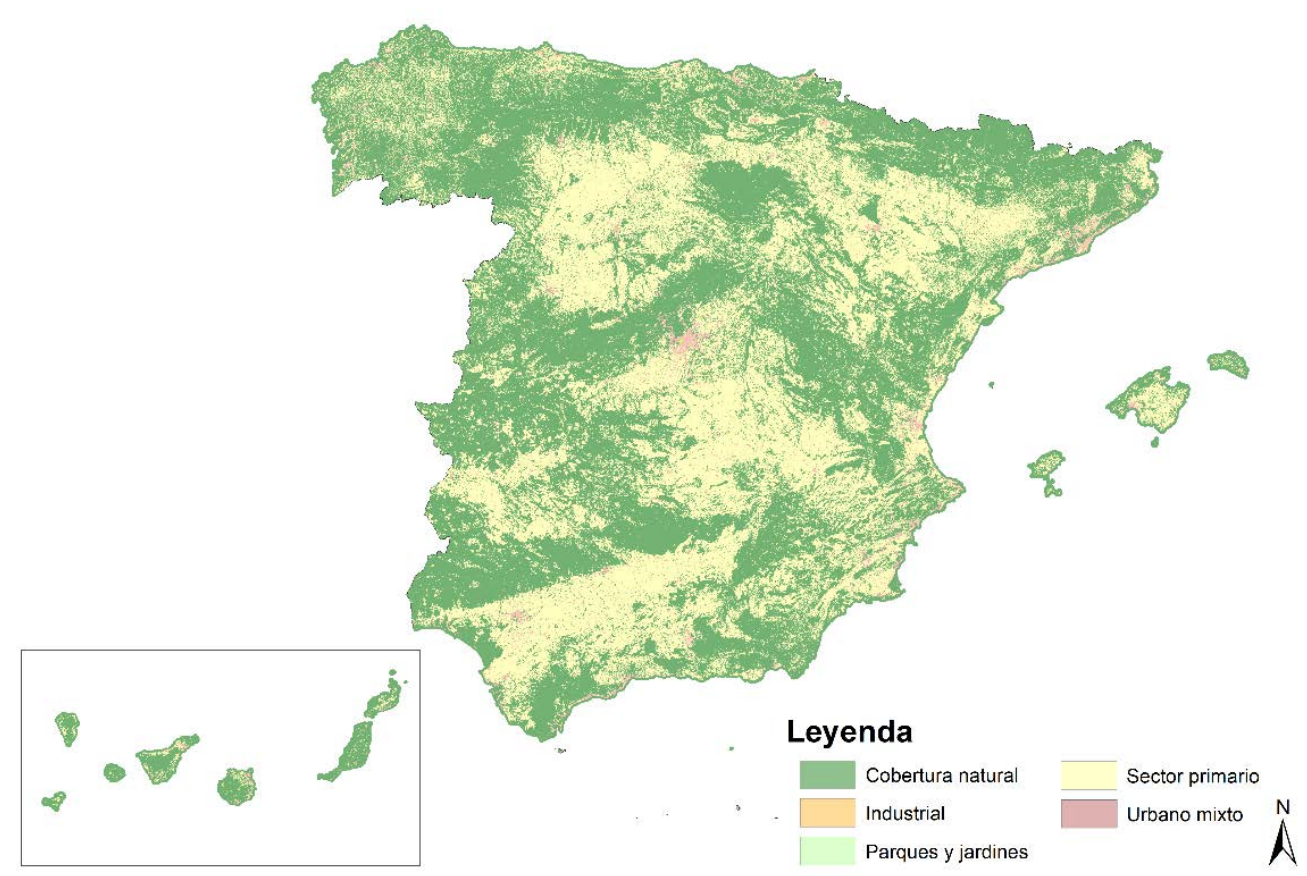

Fuente: elaboración propia a partir de cartografía obtenida del Gobierno de España

b) Zonas de Morfología Urbana (ZMU)

Se definen como un conjunto de áreas urbanas situadas entre sí a una distancia igual o inferior a 200 metros (Mielgo, 2007). Esta cartografía (Figura 3) se elabora a partir de los usos del SIOSE 2014 siguiendo los principios metodológicos de Goerlinch y Cantarino (2013) y de la Agencia Europea de Medio Ambiente para la construcción de Áreas Urbanas (Mielgo, 2007). El proceso de elaboración descansa sobre criterios morfológicos, sin referencia inicial a la demografía y sin partir de lindes administrativos, sino de los polígonos homogéneos de coberturas del suelo proporcionados por SIOSE. Para su construcción, se llevan a cabo varias operaciones con el software ArcGIS de ESRI. En primer lugar, se crea una clase que agrupa las coberturas que forman el tejido urbano (continuo y discontinuo) a partir de la cual, por criterios de distancia, se 
le agregan ordenadamente otras coberturas relacionadas con las áreas urbanas pero distintas de las anteriores; estas son zonas industriales, comercios, aeropuertos, servicios dotacionales, parques urbanos, vías de comunicación, etc. Como resultado, se obtiene una matriz urbana con huecos compuestos por coberturas no artificiales que también han de formar parte del conjunto urbano. Tras rellenar esos espacios, se eliminan de la capa resultante todas las pequeñas zonas urbanas que tengan una superficie inferior a 20 hectáreas; así, se excluyen del estudio los núcleos rurales de pequeño tamaño. En el trabajo realizado por Durán et al. (2020) puede consultarse el procedimiento de delimitación completo.

\section{Figura 3. Zonas de Morfología Urbana (ZMU)}

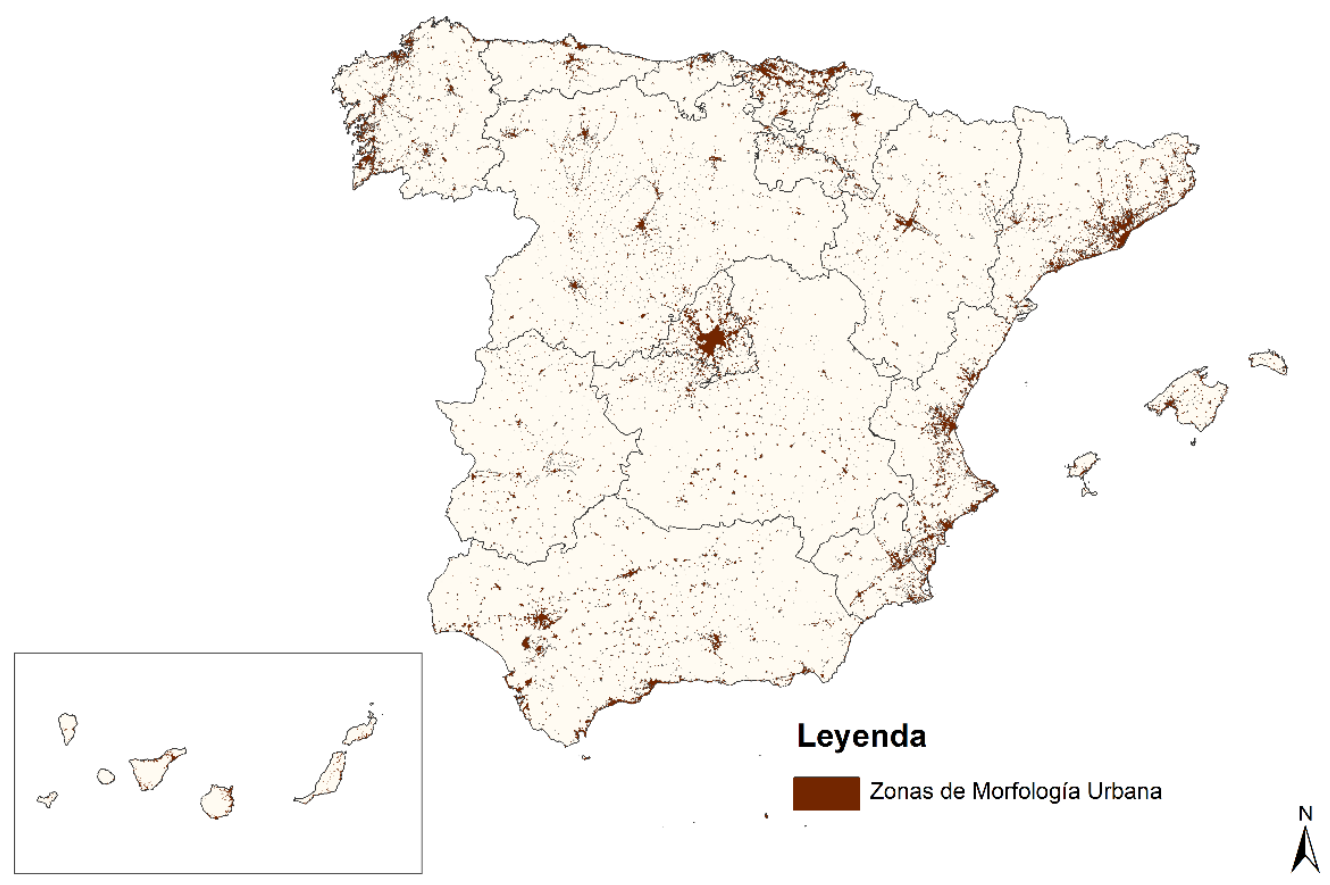

Fuente: elaboración propia a partir del SIOSE

c) Zonas inundables asociadas a períodos de retorno de diez años

Se emplean para representar, de manera genérica, el espacio fluvial. Esta cartografía muestra a escala 1:25000 las zonas que se inundan con una probabilidad del $10 \%$ anual como consecuencia del desbordamiento de los cursos de agua (Figura 4).

Se centra, sobre todo, en la representación de las zonas inundables de las áreas urbanas. Está ampliamente desarrollada para el territorio español a excepción de las Islas Canarias, donde no se han localizado mapas asociados a este período de retorno. 


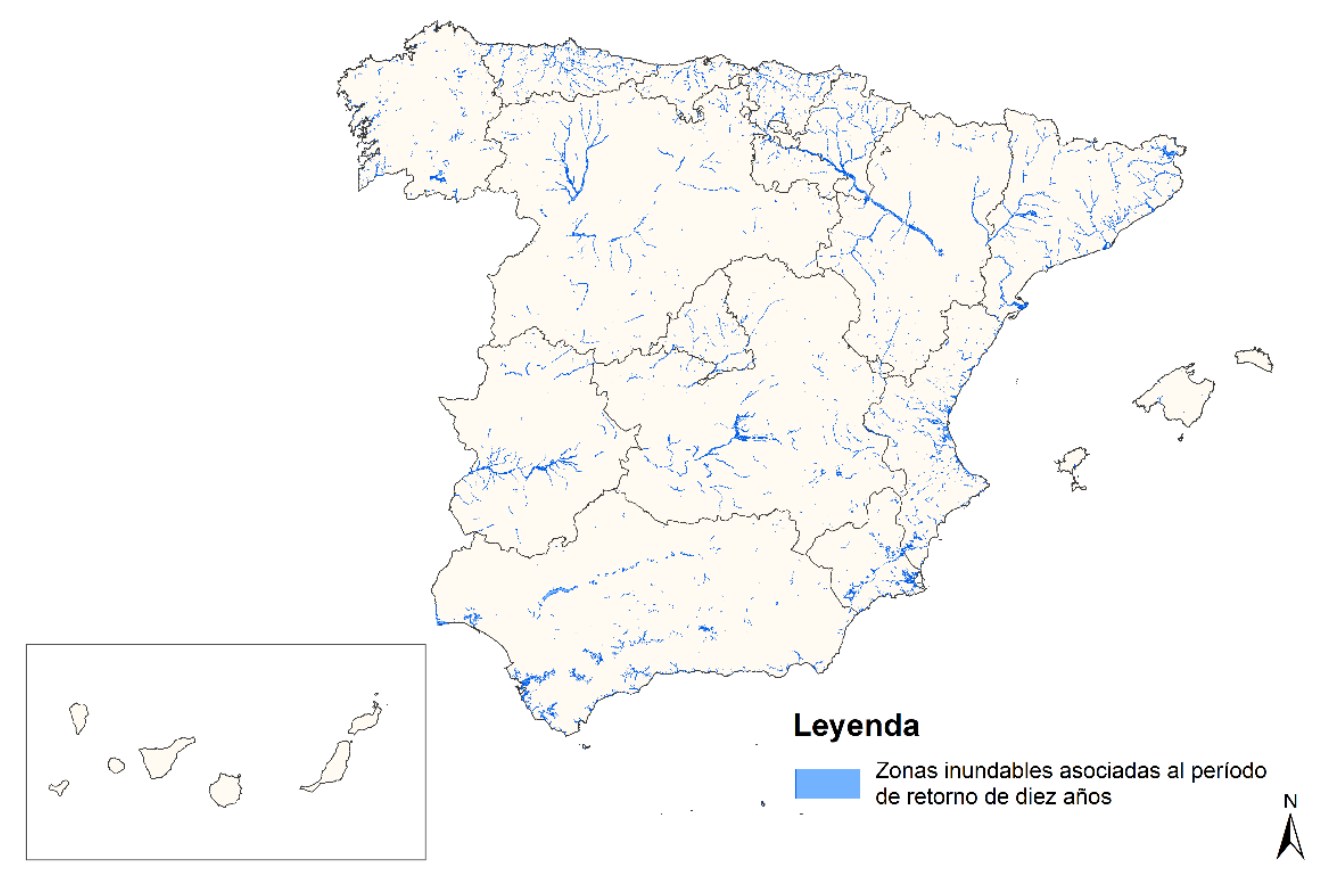

Fuente: elaboración propia a partir de cartografía de inundabilidad del Gobierno de España

d) Cartografía complementaria

A continuación, se describen los mapas empleados para realizar el análisis según las regiones climáticas y la temporalidad de flujo de los cursos de agua.

Mapa de Regiones Biogeográficas: las regiones biogeográficas muestran la extensión de las áreas con características climáticas, orográficas y geobotánicas comunes. Cuatro de las siete regiones biogeográficas establecidas en la Directiva Marco del Agua (DMA) están en España: alpina, atlántica, macaronésica y mediterránea (Figura 5).

Ríos clasificados según método Pfafstetter: Se trata de la red hidrográfica más completa disponible para toda España. Incluye la clasificación de los tramos de río de más de un kilómetro de longitud, partiendo de la cartografía 1:25000 del Instituto Geográfico Nacional (IGN) (Figura 6). Este método de clasificación es el propuesto por la Comisión Europea para codificar ríos.

Con esta cartografía se pretende distinguir entre cursos de caudal circulante de forma habitual y cursos de caudal intermitente (Doménech et al., 2008). En el lenguaje coloquial español normalmente se habla de ríos para referirse a cursos de agua cuyo caudal es continuo y existen 
otros términos que varían en función de la región para aludir a las corrientes de agua intermitentes: arroyos, rieras, errekas, regueiros, barrancos, torrentes, ramblas, etc.

Figura 5. Regiones biogeográficas

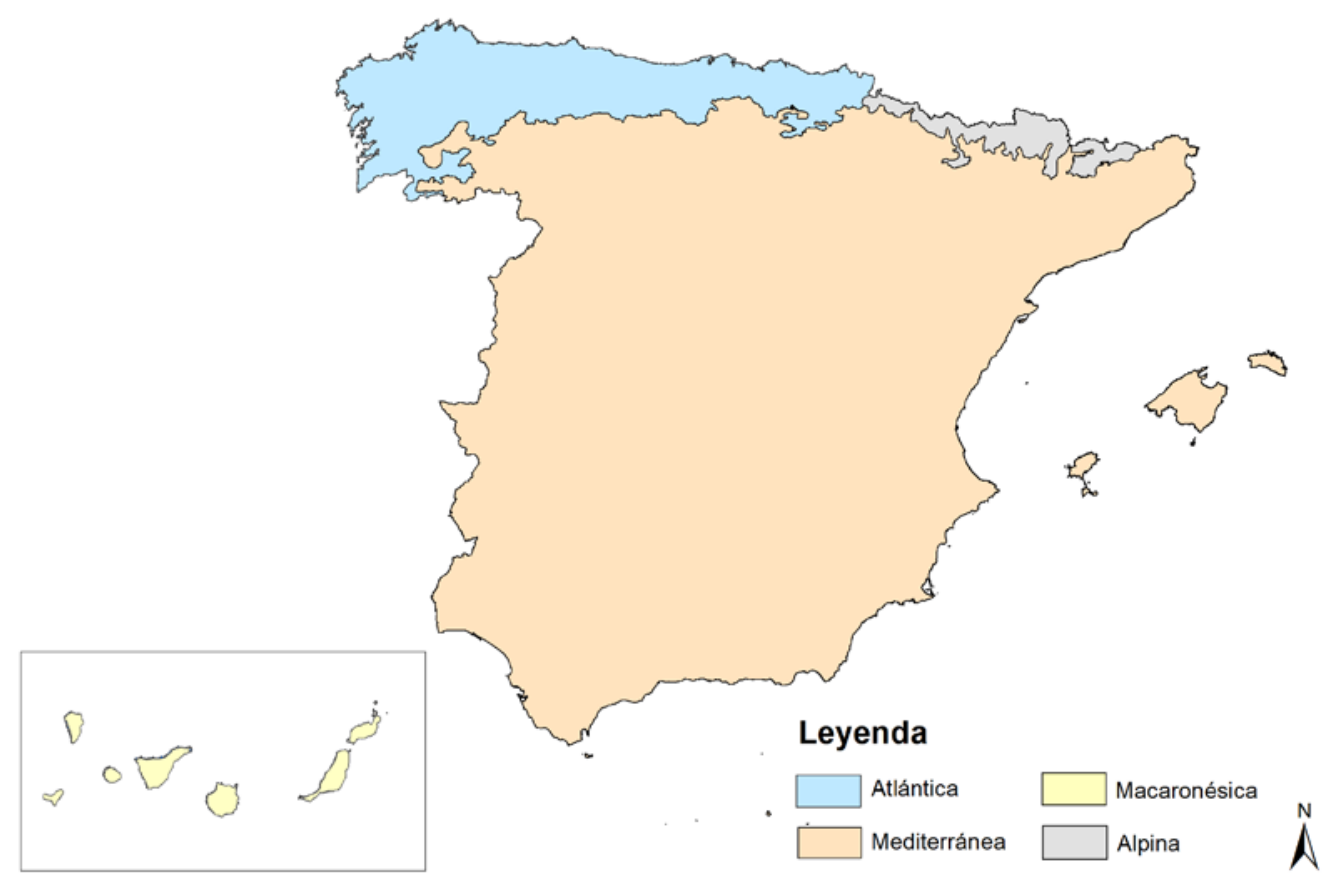

Fuente: elaboración propia a partir de cartografía de la Agencia Europea de Medio Ambiente

Puesto que uno de los campos que contiene la tabla de atributos de este mapa hace referencia al tipo de sistema fluvial, es posible diferenciar, de manera genérica, entre cursos permanentes e intermitentes. 


\section{Figura 6. Ríos clasificados según Pfafstetter}

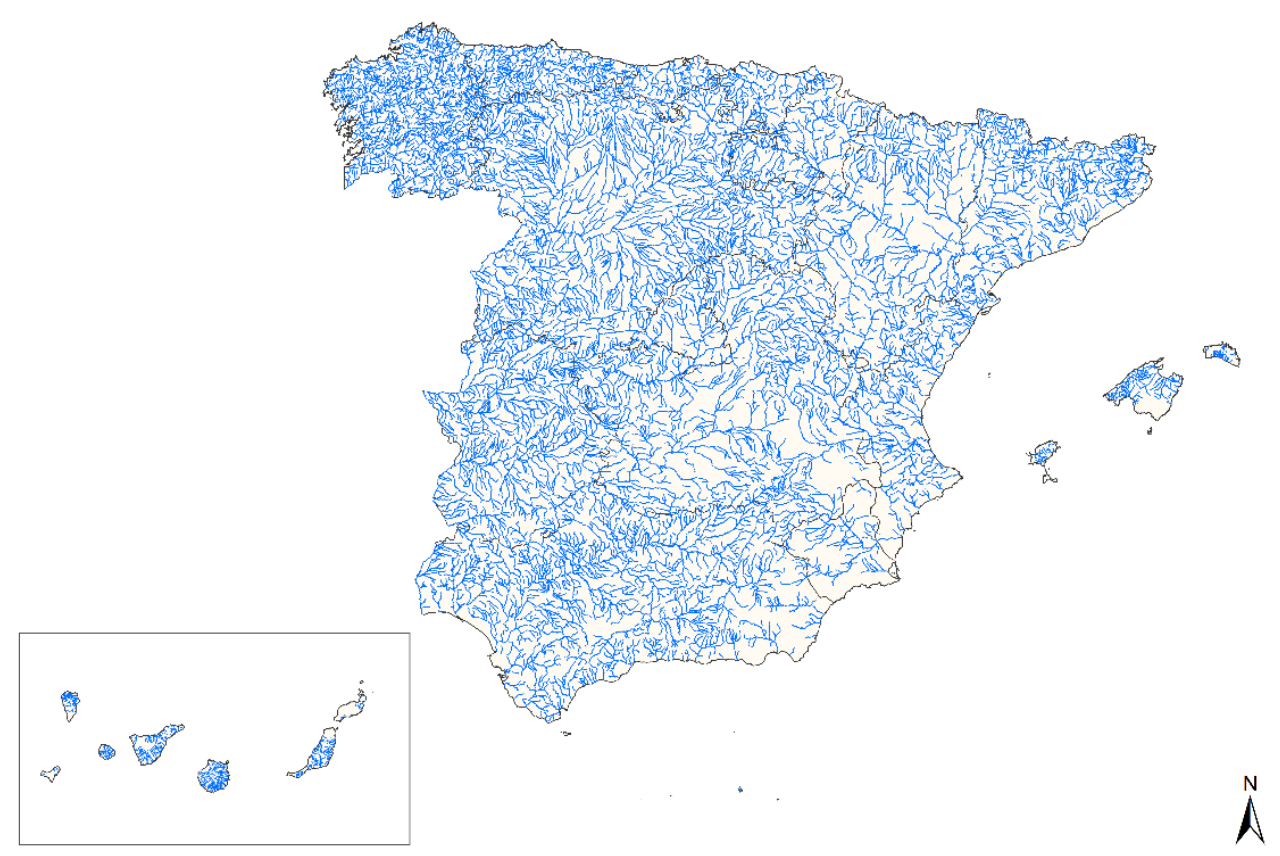

Fuente: elaboración propia a partir de cartografía hidrográfica del Gobierno de España

\subsection{Proceso constructivo}

A continuación, se explican los pasos seguidos en la creación de la capa "usos del espacio fluvial urbano".

a) Clasificación de los usos del suelo

Para la elaboración del presente estudio, se han agrupado los usos recogidos en los campos "CODIIGE" e "HILUCS" de la tabla de atributos asociada a la base de datos del SIOSE. La clasificación realizada responde a (1) la necesidad de distinguir los usos antrópicos del resto de las coberturas naturales; (2) la oportunidad de estimar la superficie dedicada a residencias en la zona inundable y (3) la conveniencia de identificar los usos compatibles y no compatibles con las inundaciones. Por otro lado, el agrupamiento permite resumir la información de la tabla y representarla de una forma más sencilla.

En el apartado introductorio se ha explicado la evolución global de los usos antrópicos en el llano de inundación. Este marco de referencia, junto con las propias observaciones de los autores, sugiere qué agrupamientos de usos pueden ser más coherentes y, además, tener un mayor interés científico-técnico. 
En primer lugar, se han ordenado los usos del suelo distinguiendo tres grupos (Tabla 1). El primero no se emplea en el presente trabajo; sin embargo, resulta útil para la realización del segundo, que distingue entre coberturas naturales y usos antrópicos. El tercer agrupamiento reconoce, de manera genérica, los usos que pueden considerarse compatibles con las inundaciones.

Tabla 1. Clasificaciones de los usos de suelo del SIOSE

\begin{tabular}{|c|c|c|c|}
\hline $\begin{array}{c}\text { Tercer } \\
\text { agrupamiento }\end{array}$ & $\begin{array}{c}\text { Segundo } \\
\text { agrupamiento }\end{array}$ & $\begin{array}{c}\text { Primer } \\
\text { agrupamiento }\end{array}$ & $\begin{array}{l}\text { Categoría en base de datos original } \\
\text { (Tabla CODIIGE del SIOSE) }\end{array}$ \\
\hline \multirow{8}{*}{$\begin{array}{c}\text { Compatible con } \\
\text { las inundaciones } \\
\text { (excepto } \\
\text { categorías } 121 \text { y } \\
122 \text { ) }\end{array}$} & \multirow{6}{*}{ Cobertura natural } & $\begin{array}{l}\text { Coberturas de } \\
\text { agua }\end{array}$ & $\begin{array}{c}\text { Curso de agua (511), lago o laguna (512), mar (515), } \\
\text { embalse (513) }\end{array}$ \\
\hline & & $\begin{array}{l}\text { Coberturas } \\
\text { húmedas }\end{array}$ & $\begin{array}{l}\text { Zona húmeda y pantanosa (411), turbera (412), marisma } \\
\text { (413), salina (414) }\end{array}$ \\
\hline & & Arbolado forestal & $\begin{array}{c}\text { Bosque de frondosas (311), bosque mixto (313), bosque } \\
\text { de coníferas (312) }\end{array}$ \\
\hline & & Matorrales & Matorral (330), combinación de vegetación (340) \\
\hline & & Pastizales & Pastizal o herbazal (320) \\
\hline & & $\begin{array}{l}\text { Terrenos sin } \\
\text { vegetación }\end{array}$ & $\begin{array}{l}\text { Playa, Duna o Arenal (351), roquedo (352), } \\
\text { temporalmente desarbolado por incendios (353), suelo } \\
\text { desnudo (354), glaciar y/o nieve perpetua (516) }\end{array}$ \\
\hline & Sector primario & Sector primario & $\begin{array}{l}\text { Instalación agrícola ganadera/ ganadera (121), } \\
\text { instalación forestal (122), extracción minera (123), } \\
\text { asentamiento agrícola y huerta (150), cultivo herbáceo } \\
\text { (210), invernadero (220), frutal no cítrico (232), frutal } \\
\text { cítrico (231), viñedo (233), olivar (234), otros cultivos } \\
\text { leñosos (235), combinación de cultivos leñosos (236), } \\
\text { prados (240), combinación de cultivos (250), } \\
\text { combinación de cultivos con vegetación (260) }\end{array}$ \\
\hline & $\begin{array}{l}\text { Parques y } \\
\text { jardines }\end{array}$ & $\begin{array}{l}\text { Parques y } \\
\text { jardines }\end{array}$ & Zona Verde Urbana (114), lámina de agua artificial (514) \\
\hline \multirow{4}{*}{$\begin{array}{c}\text { No compatible } \\
\text { con las } \\
\text { inundaciones } \\
\text { (incluidas } \\
\text { categorías } 121 \text { y } \\
122 \text { ) }\end{array}$} & \multirow{3}{*}{ Urbano mixto } & Urbano mixto & Casco (111), ensanche (112), discontinuo (113) \\
\hline & & $\begin{array}{l}\text { Infraestructuras } \\
\text { de transporte y } \\
\text { otras }\end{array}$ & $\begin{array}{c}\text { Red viaria y ferroviaria (161), puerto (162), aeropuerto } \\
\text { (163), infraestructuras de suministro (171), infraestructura } \\
\text { de residuos (172) }\end{array}$ \\
\hline & & $\begin{array}{c}\text { Equipamientos y } \\
\text { dotaciones }\end{array}$ & Servicio dotacional (140) \\
\hline & Industrial & Industrial & Industrial (130) \\
\hline
\end{tabular}

Fuente: elaboración propia

Posteriormente, se han identificado los polígonos de uso residencial según el campo "HILUCS" de la tabla SIOSE (Tabla 2). Este cuarto y último agrupamiento permite conocer la superficie residencial ${ }^{2}$ presente en las zonas inundables urbanas.

2 Esta clase está constituida por las superficies en las que dominan las residencias 


\section{Tabla 2. Clasificación según uso residencial}

\begin{tabular}{|c|c|}
\hline Cuarto agrupamiento & Categoría en base de datos original (Tabla HILUCS del SIOSE) \\
\hline & Agriculture (110), Forestry (120), Mining and Quarrying (130), Aquaculture and \\
& Fishing (140), Secondary Production (200), Commercial Services, Community \\
Non Residential Use & $\begin{array}{c}\text { Services (330), Cultural Entertainment and Recreational Services (340), Transport } \\
\text { Networks (410), Utilities (430), Transitional Areas (610), Abandoned Areas (620), } \\
\text { Land Areas not in other Economic Use (632), Not Known Use (660) }\end{array}$ \\
\hline Residential Use & Residential Use (500) \\
\hline
\end{tabular}

Fuente: elaboración propia

El resultado final es una capa única para todo el territorio nacional en la que están agrupadas las coberturas de suelo del SIOSE y que se puede consultar de manera independiente.

El cuadro de reparto de estos grupos, puede ser un indicador del impacto ecológico sobre las zonas inundables y del riesgo al que está expuesto el núcleo urbano. Generalmente, los usos industrial y urbano mixto son los que ocasionan un mayor impacto ambiental y provocan más repercusiones socioeconómicas tras producirse avenidas extraordinarias (Covarrubia et al., 2016). Por contra, los usos primarios agrícolas y, sobre todo, los espacios verdes urbanos se consideran más apropiados para la zona inundable (Ureña, 2002).

La distinción entre usos compatibles y no compatibles con las inundaciones, se basa en la presencia o ausencia de infraestructuras humanas que puedan suponer un obstáculo para la circulación de agua o aumenten el riesgo socioeconómico. Normalmente, la presencia de cualquier construcción y/o sistema viario, no se considera apropiada para un espacio que se anega frecuentemente.

b) Extracción de los usos del suelo de las áreas urbanas

Una vez agrupados los usos del suelo, se delimitan utilizando como mascara de recorte el mapa Zonas de Morfología Urbana (ZMU). El resultado es una capa de usos del suelo en las áreas urbanas de toda la geografía española. En la Figura 7 se representa el proceso cartográfico, utilizando como ejemplo la zona urbana de Pamplona.

Posteriormente, se localizan y exportan a una tabla Excel todos los usos contenidos en las áreas urbanas asentadas sobre uno o más sistemas fluviales. Estos datos se emplean en el proceso analítico para comparar el tipo y distribución de usos en el espacio de los ríos con el reparto de los mismos en las áreas urbanas. 


\section{Figura 7. ZMU (izquierda) y usos del suelo (derecha) en Pamplona}
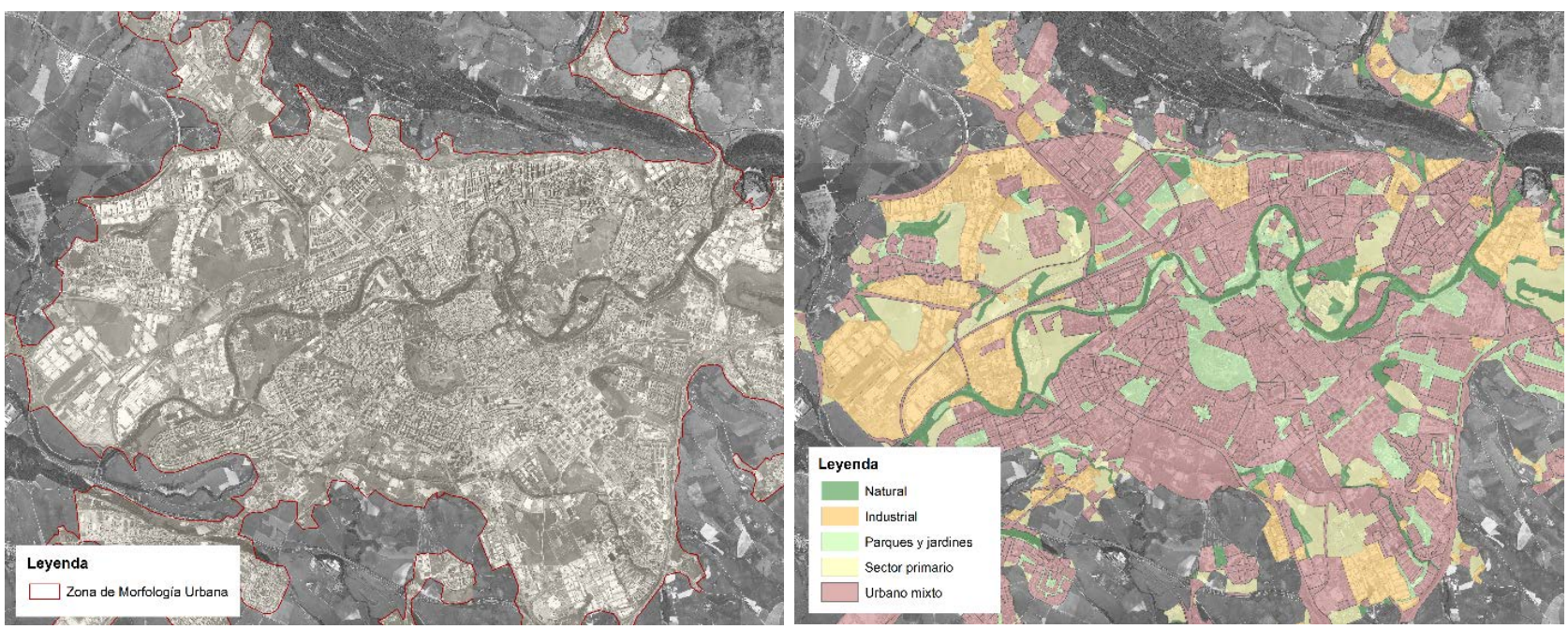

Fuente: elaboración propia a partir de cartografía hidrográfica del Gobierno de España

c) Delimitación de las zonas inundables en el ámbito urbano y extracción de usos del suelo Al igual que en el caso anterior, se utilizan las ZMU como capa de recorte para las zonas de inundación frecuente. El resultado es un mapa de los espacios inundables delimitados por las áreas urbanas del territorio español. En la Figura 8 se representa el proceso cartográfico ejemplificado con la ciudad de Pamplona.

\section{Figura 8. Delimitación del espacio fluvial en el área urbana de Pamplona}
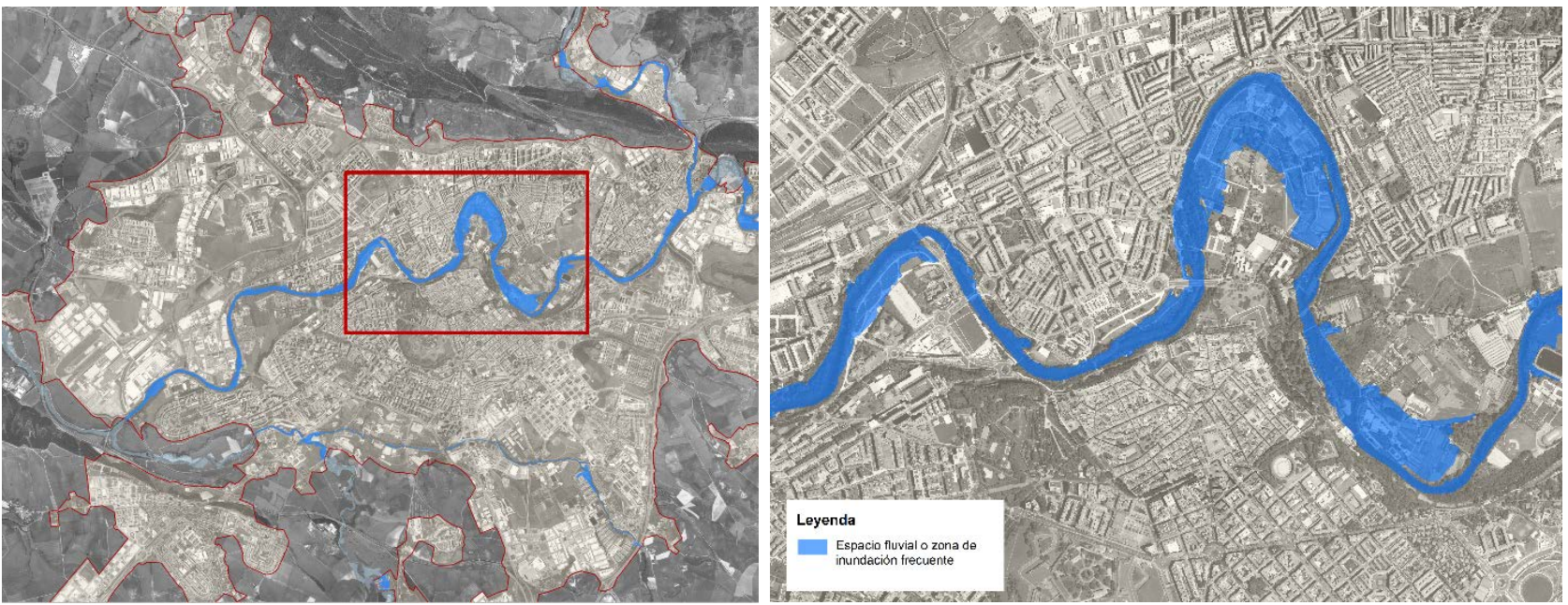

Fuente: elaboración propia a partir de cartografía hidrográfica del Gobierno de España

Posteriormente, este nuevo mapa sirve como máscara de recorte de los usos del suelo agrupados. El resultado final es el mapa de usos del suelo del espacio fluvial urbano en el que se puede representar cada agrupamiento realizado en el paso 3.2.a. En la Figura 9 se muestran, para la misma ciudad, los datos correspondientes al segundo y cuarto agrupamiento. 


\section{Figura 9. Usos del suelo en el espacio fluvial de Pamplona (izquierda)}

\section{y superficies residenciales en el río (derecha)}
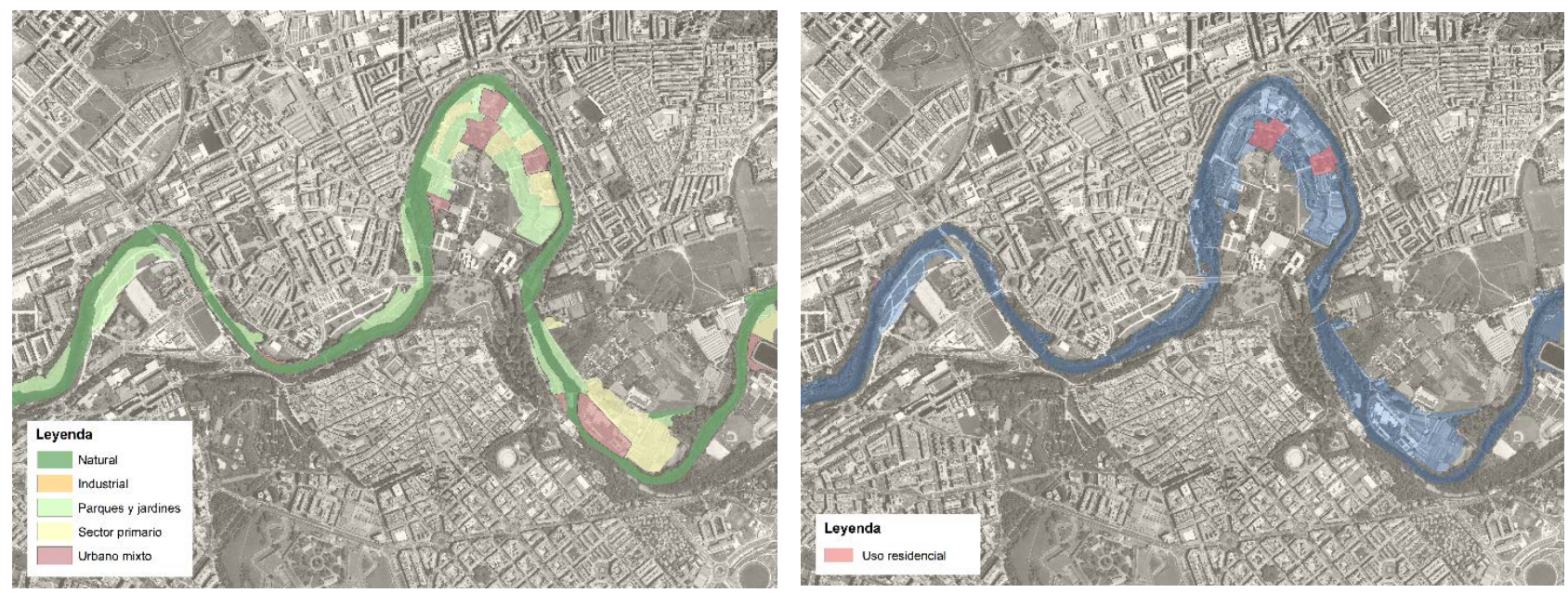

Fuente: elaboración propia a partir de cartografía hidrográfica del Gobierno de España

El proceso constructivo completo se representa en la Figura 10.

Figura 10. Creación de capa de usos del suelo del espacio fluvial urbano

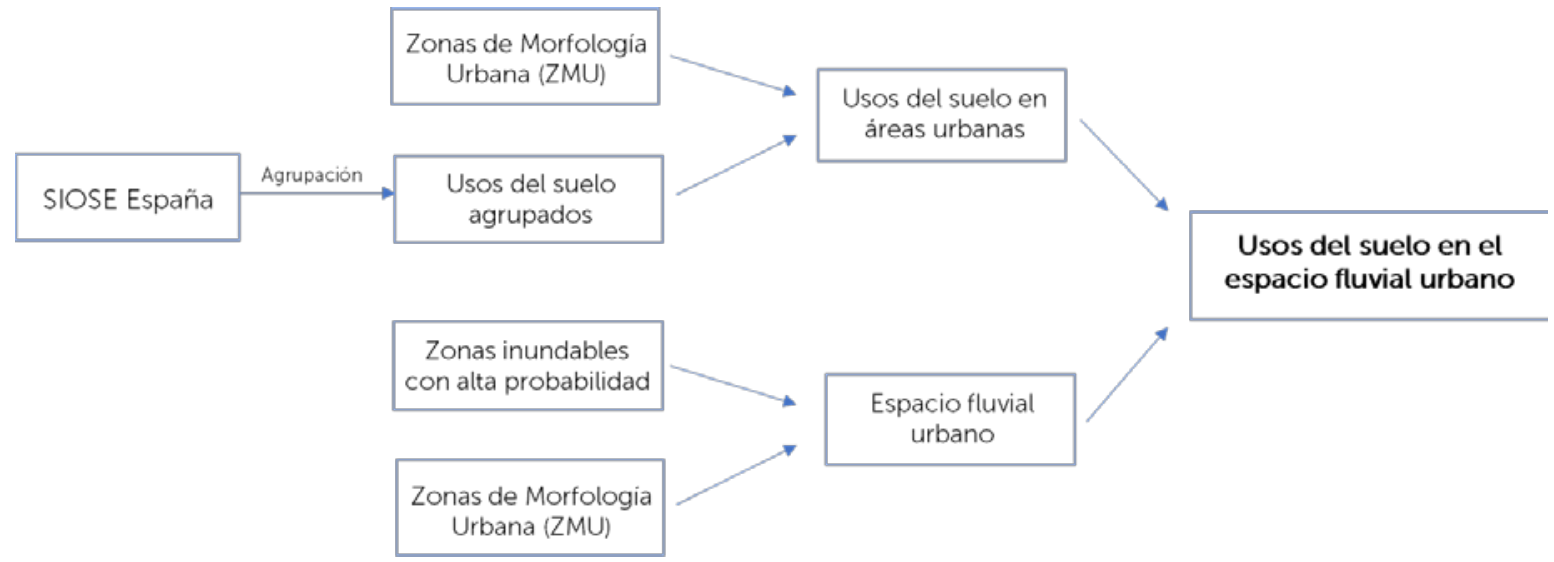

Fuente: elaboración propia

d) Asociación de los usos del suelo con las regiones biogeográficas y los ríos de España.

En este último paso del tratamiento cartográfico, se identifica cada polígono de la tabla "usos del suelo en el espacio fluvial urbano" con la región biogeográfica en la que se encuentra y con el tipo de curso de agua al que está ligado.

El procedimiento se lleva a cabo mediante la operación "selección por localización" del software ArcGIS de ESRI; así, se crearán dos nuevos campos en la tabla de atributos de la capa final: uno indica la región a la que pertenece cada polígono y otro si está asociado a un río (caudal permanente) o a otro tipo de curso (caudal no permanente). 


\subsection{Proceso analítico}

En esta fase se emplea Microsoft Excel para analizar la información contenida en las tablas de atributos asociadas a las capas "usos de las áreas urbanas fluviales" y "usos del espacio fluvial urbano". Se realizan tres comparativas de usos: espacio fluvial-área urbana, región mediterránearegión atlántica y ríos permanentes-ríos intermitentes.

\section{Resultados}

A continuación, se muestran los resultados generales y los relativos a las consultas de regiones biogeográficas y temporalidad de flujo. Los datos se representan en gráficos y tablas mediante porcentajes, refiriéndose estos últimos a la superficie total estudiada para cada análisis. Se trata de una exposición comparativa en la que se enfatiza la mayor o menor presencia de determinados usos. Solo se comentan los resultados considerados relevantes.

\subsection{Resultados generales: usos del suelo en las áreas urbanas y el espacio fluvial}

El conjunto de áreas urbanas suma una superficie de $14311 \mathrm{~km}^{2}$. El $70 \%$ de la misma está destinada a usos artificiales; es decir, urbano mixto (55\%), industrial (13\%) y parques (2\%). El $30 \%$ restante se reparte entre coberturas naturales $(9 \%)$ y usos primarios $(21 \%)$. El uso residencial ocupa una tercera parte de la superficie y los usos no compatibles con la inundación más de dos tercios del área total.

Una parte muy pequeña de toda esa superficie urbana es la que se localiza en zona inundable de origen fluvial. En concreto, se trata de $312 \mathrm{~km}^{2}$, repartidos entre 1768 municipios.

Para el caso del espacio fluvial, las coberturas naturales se triplican y los usos asociados al sector primario adquieren más protagonismo. Las superficies de urbano mixto e industrial descienden y aumenta la cantidad de espacios verdes. El suelo dedicado a uso residencial es proporcionalmente menor, ocupando un quinto del total y los usos no compatibles con las inundaciones abarcan el $44 \%$ del espacio inundable (Tabla 3).

En la Figura 11 se muestra el reparto de usos para los dos ámbitos de estudio. 
Figura 11. Porcentajes de usos del suelo en el espacio fluvial urbano

a) Espacio fluvial urbano

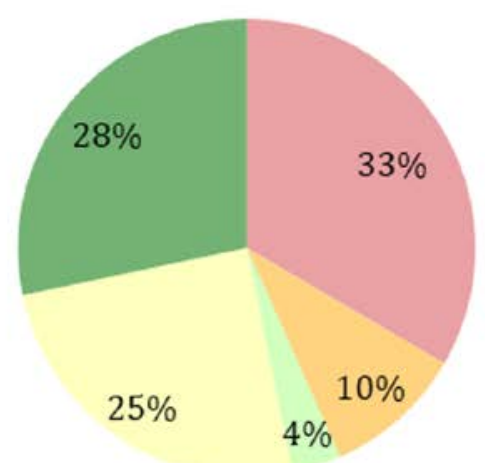

b) Áreas urbanas

\section{$9 \%$}

- Urbano mixto

= Industrial

Parques y jardines

Sector primario

natural
$21 \%$

$2 \%$

$13 \%$

Fuente: elaboración propia

Tabla 3. Porcentajes de superficie destinada a residencias y con usos no compatibles con las inundaciones

\begin{tabular}{|l|c|c|}
\hline & Uso residencial (\%) & $\begin{array}{l}\text { Superficie con usos no compatibles } \\
\text { con las inundaciones (\%) }\end{array}$ \\
\hline Espacio fluvial urbano & 18 & 44 \\
\hline Areas urbanas & 36 & 68 \\
\hline
\end{tabular}

Fuente: elaboración propia

\subsection{Resultados por regiones biogeográficas}

De los $312 \mathrm{~km}^{2}$ de zonas urbanas inundables que se han analizado, $85 \mathrm{~km}^{2}$ se encuentran en la zona atlántica y $226 \mathrm{~km}^{2}$ en la mediterránea. El estudio de la superficie de la región alpina se ha descartado debido a su baja representatividad $\left(1 \mathrm{~km}^{2}\right)$. Por otro lado, no se han obtenido datos de la región macaronésica debido a que no hay cartografía de zonas inundables asociadas al período de retorno de diez años.

Tal y como se observa en la Figura 12, los resultados de ocupación del espacio fluvial muestran una situación comparativamente más preocupante en la región mediterránea que en la atlántica. La superficie destinada al desarrollo urbano en este ámbito es 13 puntos porcentuales superior a la de la región atlántica y la cobertura natural es 11 puntos inferior. Sin embargo, y aunque sea una parte poco representativa del total, los espacios fluviales mediterráneos cuentan con el doble de suelo dedicado a zonas verdes. 
Figura 12. Porcentajes de usos de suelo en el espacio fluvial de las regiones atlántica y mediterránea

a) Región atlántica

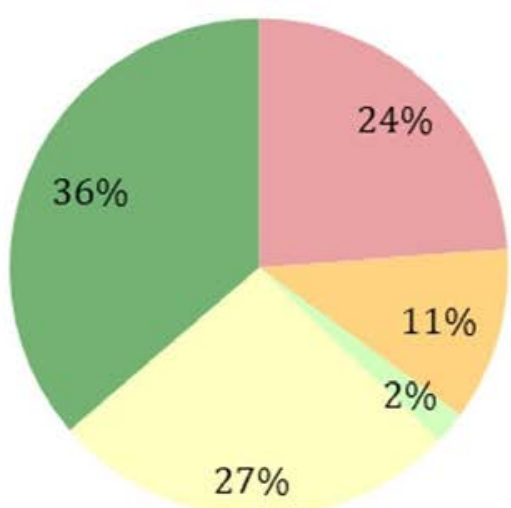

b) Región mediterránea

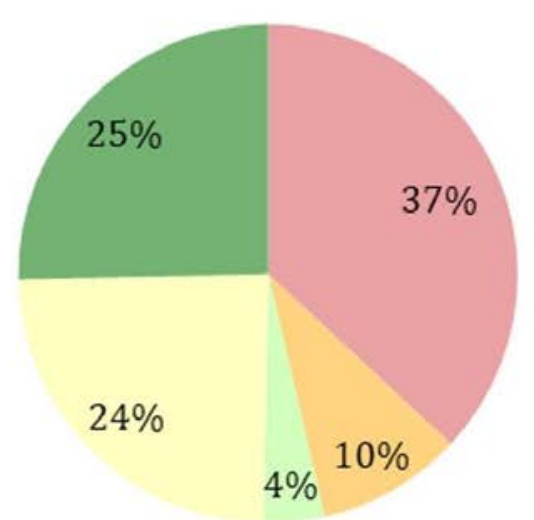

Fuente: elaboración propia

Por otro lado, tal y como se observa en la Tabla 4, tanto la ocupación residencial como la superficie destinada a usos no compatibles con las inundaciones, son superiores en la región mediterránea.

Tabla 4. Porcentajes de superficie destinada a residencias y con usos no compatibles con la inundación

\begin{tabular}{|l|c|c|}
\cline { 2 - 3 } \multicolumn{1}{c|}{} & Uso residencial (\%) & $\begin{array}{c}\text { Superficie con usos no compatibles } \\
\text { con las inundaciones (\%) }\end{array}$ \\
\hline Mediterránea & 19 & 47 \\
\hline Atlántica & 13 & 35 \\
\hline
\end{tabular}

Fuente: elaboración propia

\subsection{Resultados por temporalidad de flujo}

Por último, se ha analizado el espacio inundable urbano de un total de 922 cursos de agua. De ellos, 655 están reconocidos como ríos y 267 como arroyos, barrancos, ramblas, rieras y otros cursos intermitentes. La superficie inundable asociada a los cursos que presentan un caudal de agua continuo es de $173 \mathrm{~km}^{2}$ mientras que para los cursos estacionales, intermitentes o efímeros es de $139 \mathrm{~km}^{2}$.

Los resultados revelan una mayor ocupación antrópica de los espacios inundables asociados a los cursos intermitentes (Figura 13). En éstos, tan solo un 15\% de la superficie se mantiene natural y más de la mitad del suelo está ocupado por usos propiamente urbanos. Además, destaca la presencia del sector primario, que ocupa casi una tercera parte de la superficie total. 
En los espacios ligados a corrientes de agua continua, el uso industrial y primario están menos representados; no obstante, la presencia de parques y jardines, siendo poco representativa como en el caso anterior, aumenta considerablemente.

Figura 13. Porcentajes de usos de suelo en el espacio inundable de los cursos permanentes y no permanentes

a) Cursos de agua permanentes

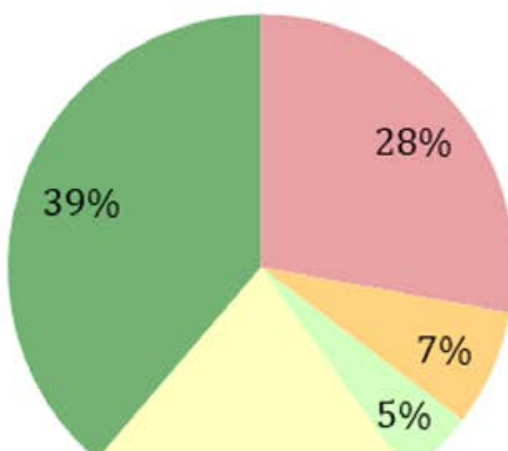

$21 \%$ b) Cursos de agua no permanentes

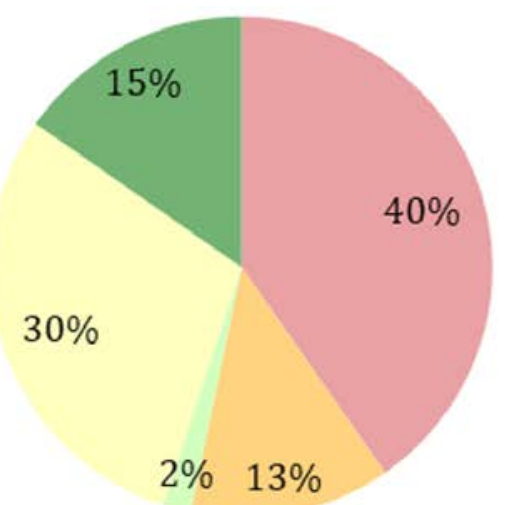

Fuente: elaboración propia

Como podría suponerse, tanto el uso residencial como los usos no compatibles con las inundaciones son superiores en el caso de los ríos intermitentes (Tabla 5). Una cuarta parte del espacio fluvial asociado a los cursos no permanentes está ocupado por residencias y más de la mitad por usos no compatibles con las inundaciones.

Tabla 5. Porcentajes de superficie destinada a residencias y con usos no compatibles con la inundación

\begin{tabular}{|l|c|c|}
\cline { 2 - 3 } \multicolumn{1}{c|}{} & Uso residencial (\%) & $\begin{array}{l}\text { Superficie con usos no compatibles } \\
\text { con las inundaciones (\%) }\end{array}$ \\
\hline Cursos de agua permanentes & 13 & 36 \\
\hline Cursos de agua no permanentes & 24 & 54 \\
\hline
\end{tabular}

Fuente: elaboración propia

\section{Discusión y conclusiones}

Este apartado se presenta dividido en dos bloques, ya que las consideraciones que se van a realizar son de muy diverso cariz, afectando tanto a las fuentes y desarrollo metodológico empleado, como a los propios resultados obtenidos en el estudio. 


\subsection{En cuanto al procedimiento metodológico}

En el presente estudio, la calidad de los resultados está condicionada, sobre todo, por la resolución de la cartografía disponible y por la falta de una delimitación precisa del espacio fluvial urbano. Es oportuno considerar estas cuestiones a la hora de interpretar los resultados.

a) Limitaciones de la cartografía empleada

El mapa de usos del suelo del SIOSE es adecuado para la escala en la que se ha trabajado. Si se compara con otras fuentes similares -CORINE Land Cover, por ejemplo- se aprecia que no solo tiene una mayor resolución espacial, sino que además permite distinguir entre muchos más usos, lo cual hace que sea especialmente apropiado en el análisis de espacios urbanos (Olazabal \& Bellet, 2017).

No obstante, el SIOSE también presenta algunos problemas. Por una parte, las diferencias metodológicas entre las comunidades autónomas a la hora de crear este producto y actualizarlo generan importantes incertidumbres cuando se pretenden obtener resultados a nivel nacional (Álvarez \& Olmedo, 2018). Por otra, al trabajar casos particulares - sobre todo en áreas urbanas de pequeño tamaño- se han observado deficiencias en la representación de las coberturas, debidas, probablemente, a la falta de criterios comunes en la fotointerpretación.

Algo similar puede afirmarse sobre la cartografía de inundabilidad, dado que parte de ella se ha elaborado siguiendo técnicas desfasadas (Menéndez-Rexach, 2015; Olcina \& Díez-Herrero, 2017). Sin embargo, a pesar de no ser muy precisa en general, su representatividad gana confianza en las áreas urbanas, pues las referencias a crecidas fluviales en estos ámbitos son abundantes desde la época romana y, por ello, el espacio inundable se conoce mejor (GarcíaCodron, 2004; Yagüe, 2010). Además, la cartografía asociada al período de retorno de diez años puede mostrar, de manera más o menos fiel, las áreas inundadas en sucesos reales (Camarasa \& Bescós, 2004); representación que empeora a medida que aumenta la magnitud de la crecida (Romero-Aloy, 2019).

En resumen, las fuentes cartográficas utilizadas tienen numerosas carencias, de las que hay que ser conscientes, para evitar cometer errores de bulto. Sin embargo, se trata de las mejores disponibles para esta escala de trabajo y, siempre que se utilicen con la debida cautela, resultan extremadamente útiles con el fin de poder hacer trabajos de carácter general.

b) Definición del espacio fluvial urbano

De acuerdo con algunos autores, el territorio de los ríos debe delimitarse conforme a criterios geomorfológicos, ecológicos e históricos, incluyendo zonas que estén dentro y fuera de la 
Ilanura de inundación (Ollero et al., 2009). No obstante, debido a lo laborioso que resultaría definir el espacio fluvial para todos los ríos del territorio nacional, en el presente trabajo se opta por representarlo mediante los mapas de inundabilidad asociados a períodos de retorno de diez años.

El uso de esta cartografía para identificar el espacio fluvial, está académicamente aceptado (Ollero et al., 2009) y se ha empleado en los ríos Allier en Francia (Bazin \& Gautier, 1996), Arga y Aragón en Navarra (Gobierno de Navarra, 2010) y Odra en Burgos (Fernández et al., 2015). Sin embargo, su uso se complementa con otros criterios que no han sido considerados en el presente trabajo. Por ello, la zona a la que se alude como espacio fluvial no es del todo exacta.

Por otro lado, debido a la urbanización, las defensas de las márgenes y la alternación de la dinámica natural de las aguas (Fernández, 2003; Ollero et al., 2009; Ollero \& Briz, 2018; González-Rojas, 2017) el espacio fluvial ha sido reducido y es complicado identificarlo. La cartografía puede mostrar superficies inundables más próximas a las naturales; es decir, al concepto "espacio fluvial", o zonas inundables que resultan de la alteración humana (Figura 14). La identificación del territorio original del río es especialmente compleja en áreas urbanas densas ubicadas aguas abajo de grandes presas (Kondolf et al., 2013); como, por ejemplo, el caso del río Manzanares en Madrid.

Para tener una imagen más aproximada del espacio fluvial habría que trabajar con mapas de inundabilidad basados en estudios geomorfológicos de ríos en régimen natural. No obstante, este tipo de estudios son poco frecuentes en España (Sánchez \& Lastra, 2011; Martínez et al., 2017). De hecho, solo en el noroeste del país se han seguido estos criterios para delimitar zonas con alta probabilidad de inundación y no se han utilizado de manera exclusiva en el presente estudio por la poca representatividad de los resultados.

En resumen, se puede afirmar que tras utilizar una cartografía "incompleta" que no considera todos los criterios que definen el espacio fluvial pero que con frecuencia asume las acciones humanas destinadas a controlar los ríos, el espacio fluvial que se representa en el presente trabajo ocupa una superficie más reducida que la propiamente natural. 
Figura 14. Espacio fluvial del Piles a su paso por Gijón (izquierda) y del Manzanares en Madrid
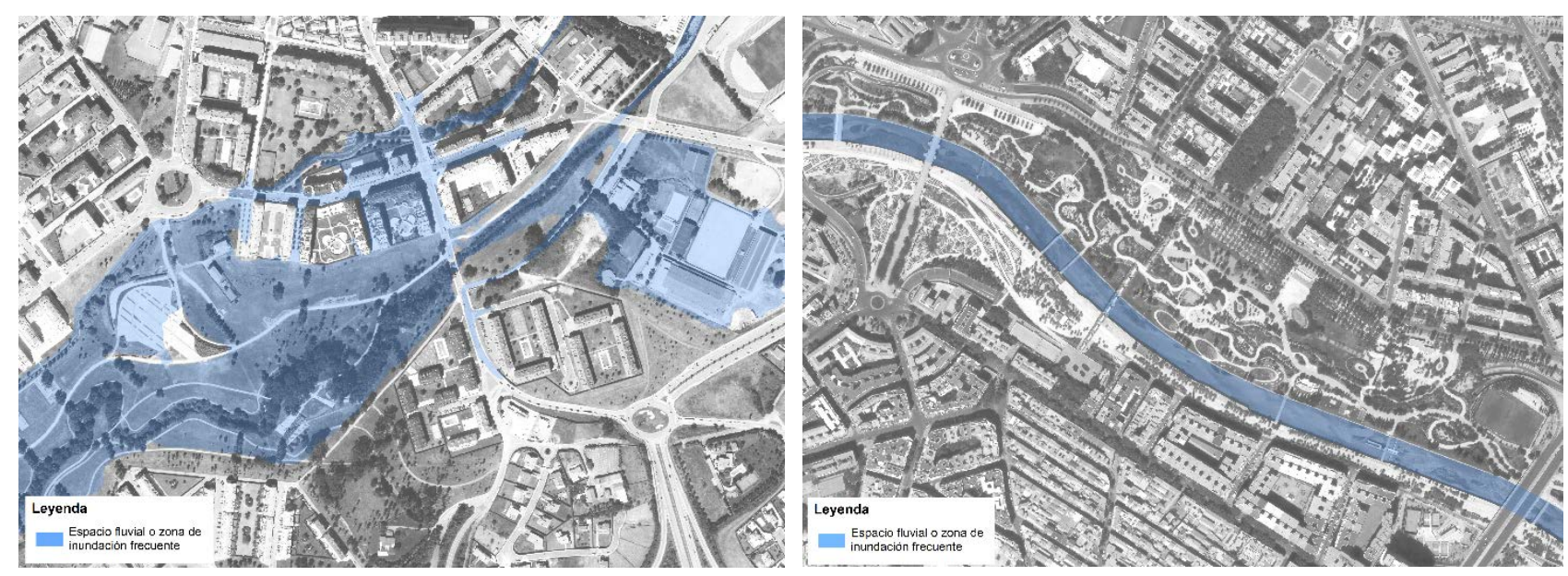

Fuente: elaboración propia a partir de cartografía del Gobierno de España

\subsection{En cuanto a los resultados}

Es esencial considerar tres cuestiones en torno a los datos obtenidos. Primera, que los resultados de ocupación del presente trabajo son fieles a la realidad de las zonas inundables urbanas pero infraestiman los datos de ocupación del espacio fluvial original. Segunda, que la escala de trabajo es limitada y, por tanto, los datos son abordables desde la generalización. Tercera, que los resultados muestran un patrón para el conjunto de casos, siendo preciso recordar que cada ámbito es único e independiente, pues las características del río y el contexto ambiental, histórico y cultural de la ciudad también influyen en el uso de la zona inundable (Gusmaroli, 2018; Santasusagna \& Tort, 2018).

En cuanto a la comparativa de usos en el territorio inundable y las áreas urbanas, se ha comprobado que los corredores fluviales en las ciudades son lugares donde se concentran numerosos espacios verdes, ya que proporcionalmente poseen más coberturas naturales, parques y zonas agrícolas y ganaderas que la media del conjunto urbano. Esta idea concuerda con diferentes estudios de ámbito internacional en los que se llega a las mismas conclusiones (Bravard, 2006; Bae, 2011; Knoll et al., 2017; Ollero \& Briz, 2018). Todo ello incluso a pesar de que los últimos proyectos de regeneración urbana hayan ocasionado una pérdida paulatina de zonas agrícolas y otros usos tradicionales (Santasusagna \& Tort, 2018). Las ciudades de Zaragoza, Pamplona, Vitoria, Logroño o Burgos son claros ejemplos en los que se observa el potencial que tienen los espacios inundables como infraestructuras verdes. 
Así mismo, los resultados muestran indicios de que la actividad industrial permanece ligada a los ríos, probablemente por la ubicación original que tuvieron algunas de las primeras instalaciones fabriles.

Resulta llamativa la elevada presencia de usos artificiales en un territorio con alta probabilidad de inundación. Para interpretar correctamente este resultado, hay que tener en cuenta las limitaciones de la cartografía de inundabilidad, pues puede considerar -en mayor o menor medida- las infraestructuras de control de crecidas fluviales. Así, en determinados casos, parte de la superficie urbana representada como inundable no se ve, de facto, anegada por las aguas (debido a la presencia de infraestructuras de control), mientras que en otros, habrá usos urbanos en zonas originalmente inundables que no están representados en el mapa. En cualquier caso, el reparto de usos en el espacio fluvial urbano, indica que se ha invadido el territorio de los ríos a su paso por las ciudades, pues casi tres cuartas partes de su superficie están ocupadas por usos antrópicos. Particularmente grave resulta el solapamiento con las zonas residenciales y las áreas dominadas por usos no compatibles con la inundación, lo que una vez más pone de manifiesto la falta de integración río-ciudad en España (Mateu, 1990; Sala \& Inbar, 1992; Pellicer, 2005; Monclús, 2002; Ureña, 2002; Fernández, 2003).

Por último, otro dato a comentar respecto a los resultados generales, es que parte de las coberturas naturales (agua, zonas húmedas, arbolado forestal, matorrales, pastizales y zonas sin vegetación) son superficies destinadas al uso recreativo que no están reconocidas como "parques" en el SIOSE. Por ello, los porcentajes referidos a parques y jardines son en realidad superiores, lo que sería más lógico en vista de los recientes esfuerzos por fomentar el uso público en las riberas urbanas.

En la comparativa por regiones biogeográficas, los datos muestran una mayor ocupación del espacio fluvial mediterráneo con respecto del atlántico, confirmando que la presión urbana en los ríos mediterráneos es muy alta (Mateu, 1990; Mateu y Camarasa, 2000; Arranz-Lozano, 2008; Conesa \& Pérez, 2014; Noguera et al., 2018; Observatorio de la Sostenibilidad, 2019). En este último caso, es especialmente preocupante la gran extensión dedicada a usos que no son compatibles con la inundación.

Por otro lado, aunque la presencia de parques y jardines es poco significativa en las áreas inundables de ambas regiones, el análisis realizado indica que, para el caso mediterráneo, la superficie destinada a este uso se duplica con respecto a la zona atlántica. Esto puede deberse, entre otras razones, a que la llanura de inundación activa es más variable en esta región (Malavoi 
\& Souchon, 1989; Mateu, 1989; EWGDF, 2010), lo que limita las posibilidades de desarrollar otros usos en esa zona que se inunda con una periodicidad alta.

En cuanto a la comparativa por temporalidad de flujo o tipología fluvial, los resultados revelan que la ocupación - tanto por usos urbanos como agrícolas- es significativamente mayor en los cursos temporales. Se confirman así las ideas recogidas por varios autores de que la actividad agrícola destaca en este tipo de ríos (Camarasa et al., 2018) y que son ecosistemas muy presionados a escala local por el ser humano (Olcina, 2004; Doménech et al., 2008; Noguera et al., 2008; Kondolf et al., 2013), siendo abrumadores los datos de ocupación residencial y de superficies con usos incompatibles con la inundación.

Por otro lado, se constata que la presencia de agua es un factor atrayente para el uso público (Hussein, 2006), pues la superficie de parques y jardines se duplica en los espacios fluviales por los que circula agua de manera habitual.

Resumiendo los resultados de los tres análisis, se observa que el espacio fluvial en las áreas urbanas ha sido ampliamente ocupado por usos antrópicos, conservando solo un tercio de su superficie natural, en particular en los ríos de la región mediterránea y, sobre todo, en los cursos de caudal intermitente (pertenezcan o no a la región mediterránea). A pesar de ello, la abundante presencia de coberturas naturales, espacios ajardinados y áreas agrícolas y ganaderas en las riberas urbanas de los ríos los convierte en potenciales infraestructuras verdes a su paso por las ciudades.

Las diferencias de ocupación observadas en las consultas por regiones biogeográficas y período de actividad fluvial, pueden responder a múltiples factores de origen geográfico, histórico y/o cultural. No obstante, algunos estudios (Camarasa \& Soriano, 2008; Doménech et al., 2008; EWGDF, 2010; Kondolf et al., 2013) señalan la posible influencia de las características de los ríos mediterráneos en la ocupación de los mismos. Las crecidas rápidas, fuertes e irregulares, generan una zona inundable extensa y poco advertida que puede favorecer el asentamiento. Además, es importante considerar que el espacio de los cursos pequeños e intermitentes ha sido muy reducido mediante canalizaciones y, en el peor de los casos, soterramientos (Elmore \& Kaushal, 2008; Kondolf et al., 2013). Así, aquellos mapas elaborados sin considerar estas intervenciones, no siempre son certeros en la representación de las verdaderas zonas inundables.

Como reflexión, este trabajo pone de manifiesto la falta de consideración del espacio inundable de los ríos en los modelos de ordenación territorial y planificación urbana (Olcina, 2004; 
Doménech et al., 2008; Herrera, 2014) y deja constancia de la necesidad de una adecuada planificación de los usos del suelo en el ámbito urbano como medida no estructural para evitar daños por avenidas fluviales (Ureña, 2002; Ollero et al., 2009; Berga-Casafont, 2011; Conesa \& Pérez, 2014; Fernández et al., 2015; Vázquez-Rodriguez, 2017; Amoateng et al., 2019). En dicha planificación, debe prevalecer la idea de conservar intacto el espacio fluvial para que éste disipe la energía de las aguas de crecida y reduzca así su peligrosidad (Mateu, 1990; Ollero et al., 2009; Ollero \& Briz, 2018).

Para concluir, puede intuirse -a pesar de no haber considerado el grado de alteración geomorfológico y dinámico de los propios ríos- que existe un impacto ambiental sobre las riberas urbanas españolas derivado de la urbanización. Por otro lado, se deduce - sin haber integrado en el estudio variables relacionadas con la peligrosidad tales como el calado o la velocidad del agua- que la probabilidad de que haya consecuencias socioeconómicas asociadas a las avenidas fluviales es alta; pues el riesgo es muy elevado. Este estudio no profundiza sobre estas cuestiones pero puede ser útil para contribuir a una sistematización metodológica que permita ampliar y contrastar el conocimiento de esta línea científica. Además, este tipo de trabajos pueden tener una relevancia en el ámbito de la planificación y gestión urbanística de los espacios fluviales.

Declaración responsable: Las/os autoras/es declaran que no existe ningún conflicto de interés con relación a la publicación de este artículo. Ambos autores han llevado a cabo la revisión bibliográfica oportuna así como la redacción del trabajo. 


\section{Bibliografía}

Adikari, Y., \& Yoshitani, J. (2009). Global trends in water-related disasters: an insight for policymakers. United Nations World Water Assessment Programme Third World Water Development, International Centre for Water Hazard and Risk Management (ICHARM) and UNESCO. Retrieved from https://www.preventionweb.net/publications/view/17742

Akter, T., Quevauviller, P., Eisenreich, S. J., \& Vaes, G. (2018). Impacts of climate and land use changes on flood risk management for the Schijn River, Belgium. Environmental science \& policy, 89, 163-175. https://doi.org/10.1016/j.envsci.2018.07.002

Alvarez, D. G., \& Olmedo, M. T. C. (2018). Caracterización de SIOSE. Revisión de la utilidad e incertidumbre de esta base de datos. In Tecnologías de la información geográfica: perspectivas multidisciplinares en la sociedad del conocimiento (pp. 928-937). Universitat de València.

Amoateng, P., Finlayson, C. M., Howard, J., \& Wilson, B. (2018). A multi-faceted analysis of annual flood incidences in Kumasi, Ghana. International journal of disaster risk reduction, 27, 105 117. https://doi.org/10.1016/j.ijdrr.2017.09.044

Arranz Lozano, M. (2008). El riesgo de inundaciones y la vulnerabilidad en áreas urbanas. Análisis de casos en España. Estudios geográficos, 69(265), 385416. https://doi.org/10.3989/estgeogr.0417

Asociación de Geógrafos Españoles \& Colegio de Geógrafos (2006). Manifiesto por una nueva cultura del territorio. Madrid. Retrieved from http://age.ieg.csic.es/docs_externos/06-05manifiesto_cultura_territorio.pdf

Ayala-Carcedo, F. J. (2002). El sofisma de la imprevisibilidad de las inundaciones y la responsabilidad social de los expertos. Un análisis del caso español y sus alternativas. Boletín de la Asociación de Geógrafos Españoles, (33). Retrieved from https://bage.agegeografia.es/ojs/index.php/bage/article/view/416

Bae, H. (2011). Urban stream restoration in Korea: Design considerations and residents' willingness to pay. Urban Forestry \& Urban Greening, 10(2), 119-126.

Baena, R. (2006). Los ríos como oportunidad para la recuperación del patrimonio natural de las ciudades desde la cooperación institucional. In R. Baena, I. Guerrero, J. C. Posada \& E. López, Ríos y ciudades europeas. Espacios naturales, culturales y productivos (pp. 37-43). Sevilla: Universidad de Sevilla, Sevilla. 
Baena, R., Guerrero, I., García, B., \& Posada, J. C. (2016). Ocupación antrópica y riesgos de inundación en la llanura del río Guadalquivir (sector Palma del Río-Sevilla, España). Boletín de la Asociación de Geógrafos Españoles, 72, 149-167. https://doi.org/10.21138/bage.2335

Ballais, J. L., Chave, S., Dupont, N., Masson, É., \& Penven, M. J. (2011). La méthode hydrogéomorphologique de détermination des zones inondables. Physio-Géo, 5. https://doi.org/10.4000/physio-geo.3307

Baptist, M. J., Penning, W. E., Duel, H., Smits, A. J., Geerling, G. W., Van der Lee, G. E., \& Van Alphen, J. S. (2004). Assessment of the effects of cyclic floodplain rejuvenation on flood levels and biodiversity along the Rhine River. River Research and Applications, 20(3), 285-297. Retrieved from https://onlinelibrary.wiley.com/doi/10.1002/rra.778

Barriendos, M., Gil-Guirado, S., Pino, D., Tuset, J., Pérez-Morales, A., Alberola, A., Costa, J., ... Ruiz-Bellet, J. L. (2019). Climatic and social factors behind the Spanish Mediterranean flood event chronologies from documentary sources (14th-20th centuries). Global and Planetary Change, 182, 102997. https://doi.org/10.1016/j.gloplacha.2019.102997

Bazin, P., \& Gautier, E. (1996). Un espace de liberté pour la Loire et l'Allier: de la détermination géomorphologique à la gestion. Revue de Géographie de Lyon, 71(4), 377-386.

Berga-Casafont, L. (2003). Presas y embalses en la España del siglo XX. Revista de Obras Públicas 3438, 37-40.

Berga-Casafont, L. (2011). Las inundaciones en España. La nueva Directiva Europea de inundaciones. Revista de Obras Públicas, 3520, 158, 7-18.

Biron, P., Buffin-Bélanger, T., Larocque, M., Choné, G., Cloutier, C.A., Ouellet, M.A., Demers, S., Olsen, T., Desjarlais, C., \& Eyquem, J. (2014). Freedom space for rivers: a sustainable management approach to enhance river resilience. Environmental management, 54(5), 10561073. https://doi.org/10.1007/s00267-014-0366-z"10.1007/s00267-014-0366-z

Buijse, A. D., Coops, H., Staras, M., Jans, L. H., Van Geest, G. J., Grift, R. E., ... Roozen, F. C. (2002). Restoration strategies for river floodplains along large lowland rivers in Europe. Freshwater biology, 47(4), 889-907.

Burriel, E.L. (2008). La "década prodigiosa" del urbanismo español (1997-2006). Scripta Nova, 12. Retrieved from http://www.ub.edu/geocrit/sn/sn-270/sn-270-64.htm 
Bravard, J. P. (2006). Rivers as cultural and meeting points some reasons for success. In Ríos y Ciudades Europeas: Espacios Naturales, Culturales y Productivos (pp. 27-35). Sevilla: Secretariado de Publicaciones de la Universidad de Sevilla.

Camarasa, A. M. (2016). Flash floods in Mediterranean ephemeral streams in Valencia Region (Spain). Journal of hydrology, 541, 99-115. https://doi.org/10.1016/j.jhydrol.2016.03.019

Camarasa, A. M., Caballero López, Ma P., \& Iranzo García, E. (2018). Cambios de uso del suelo, producción de escorrentía y pérdida de suelo. Sinergias y compensanciones en una rambla mediterránea (Barranc del Carraixet, 1956-2011). Boletín de la Asociación de Geógrafos Españoles, (78), 127-153. http://dx.doi.org/10.21138/bage.2714

Camarasa, A.M., \& Bescós, A. (2004). Cartografía de áreas inundables: comparación entre mapas de peligro y mapas de inundaciones concretas. Riesgos Naturales y Antrópicos en Geomorfología, 2, 25-36.

Camarasa, A. M., \& Soriano, J. (2008). Peligro, vulnerabilidad y riesgo de inundación en ramblas mediterráneas: los llanos de Carraixet y Poyo. Cuadernos de geografía, (83), 1-26.

Conesa García, C., \& Pérez Cutillas, P. (2014). Alteraciones geomorfológicas recientes en los sistemas fluviales mediterráneos de la Península Ibérica: Síntomas y problemas de incisión en los cauces. Revista de Geografía Norte Grande, (59), 25-44.

Covarrubia, J. C., Rayburg, S., \& Neave, M. (2016). The influence of local land use on the water quality of urban rivers. International Journal of GEOMATE, 11(23), 2155 2161. http://dx.doi.org/10.21660/2016.23.1187

Carré, C., \& Deutsch, J. C. (2015). L'eau dans la ville: une amie qui nous fait la guerre, Paris, Éditions de l'Aube.

Dewan, A. M., \& Yamaguchi, Y. (2009). Land use and land cover change in Greater Dhaka, Bangladesh: Using remote sensing to promote sustainable urbanization. Applied geography, 29(3), 390-401.

Doménech, S., Ollero, A., \& Sánchez, M. (2008). Núcleos de población en riesgo de inundación fluvial en Aragón: diagnóstico y evaluación para la ordenación del territorio. Geographicalia, (54), 17-44.

Durán, F., Pons, J.J, \& Serrano, M. (2020). ¿ Qué es un río urbano? Propuesta metodológica para su delimitación en España. ACE: Architecture, City and Environment, 15(44), 9035. http://dx.doi.org/10.5821/ace.15.44.9035 
Durán, Z. V. H., Rodríguez, P. C. R., Francia, M. J. R., \& Martín, P. F. J. (2013). Land use changes in a small watershed in the Mediterranean landscape (SE Spain): environmental implications of a shift towards subtropical crops. Journal of Land Use Science, 1, 3758. http://dx.doi.org/10.1080/1747423X.2011.620992

Elmore, A. J., \& Kaushal, S. S. (2008). Disappearing headwaters: patterns of stream burial due to urbanization. Frontiers in Ecology and the Environment, 6(6), 308-312.

Entwistle, N. S., Heritage, G. L., Schofield, L. A., \& Williamson, R. J. (2019). Recent changes to floodplain character and functionality in England. Catena, 174, 490498. https://doi.org/10.1016/j.catena.2018.11.018

Estado Español (2008). Orden ARM/2656/2008, de 10 de septiembre, por la que se aprueba la instrucción de planificación hidrológica. Boletín Oficial del Estado, 229, 38472-38582. Retrieved from https://www.boe.es/diario_boe/txt.php?id=BOE-A-2008-15340

European Environmental Agency (2018). Why should we care about floodplains? Water and $\begin{array}{llll}\text { marine } & \text { Environment. } & \text { freshwater. }\end{array}$ from https://www.eea.europa.eu/themes/water/european-waters/why-should-we-care-aboutfloodplains

European Working Group on Dams and Floods (2010). Dams and floods in Europe, Role of dams in flood mitigation. In L. Berga (Ed.), 8th ICOLD European Club Symposium.

Fernández, C. E. R. (2003). Ponferrada y el río Sil: el reencuentro de una ciudad con su origen. In La ciudad: nuevos procesos, nuevas respuestas (pp. 287-296). Secretariado de Publicaciones y Medios Audiovisuales.

Fernández, D., Robledo, M., Bielsa, J., \& Ollero, A. (2015). Análisis coste-eficacia para una propuesta de implantación del territorio fluvial en el río Odra (Burgos). In II Congreso Ibérico de Restauración Fluvial. Pamplona.

Gallegos-Reina, A. (2017). Análisis de los cambios legislativos recientes en materia de dominio público hidráulico e inundabilidad en la legislación estatal: valoraciones para la gestión territorial. En Naturaleza, territorio y ciudad en un mundo global. In XXV Congreso de la Asociación de Geógrafos Españoles (pp. 2471-2479). Madrid.

García-Codron, J. C. (2004). Las ciudades españolas y el riesgo de inundación: permanencia y cambio de un problema crónico. Boletín de la Asociación de Geógrafos Españoles, (37), 85-89. Retrieved from https://bage.age-geografia.es/ojs/index.php/bage/article/view/1978/0 
García-Codron, J. C., Garmendia-Pedraja, C., \& Rasilla, D. (2012). Avenidas e inundaciones históricas en el Cantábrico: factores climáticos y cambios en el tiempo. Publicaciones de la Asociación Española de Climatología. Serie A; 8.

Gobierno de Navarra (2010). Estudio de alternativas de actuación de restauración de ríos y defensa frente a inundaciones en la confluencia del Arga-Aragón: Plan de restauración ecológica (Unpublished report).

Goerlich, F. J., \& Cantarino, M. I. (2013). Zonas de morfología urbana: coberturas del suelo y demografía. Bilbao: Fundación BBVA. Retrieved from http://hdl.handle.net/10550/58272

González, M. A., De la Lastra, I., \& Rodríguez, I. (2007). La urbanización y su efecto en los ríos. Mesas de trabajo de la Estrategia Nacional de Restauración de ríos. Madrid: Ministerio de Medio Ambiente y Universidad Politécnica de Madrid.

González Rojas, D. (2017). La pérdida de espacios fluviales en Andalucía (1956-2007). Una aproximación escalar. Revista de Estudios Andaluces, 34(1), 2651. http://dx.doi.org/10.12795/rea.2017.i34.02

Grantham, T. E., Merenlender, A. M., \& Resh, V. H. (2010). Climatic influences and anthropogenic stressors: an integrated framework for streamflow management in Mediterraneanclimate California, USA. Freshwater Biology, 55, 188-204.

Gusmaroli, G. (2018). Urban River Restoration. X Aniversario de la Exposición Internacional 2008. Zaragoza, June 14th.

Hernández, N. C. (2018). El río y su territorio. Espacio de libertad: un concepto de gestión. Terra Nueva Etapa, 34(56). https://www.redalyc.org/articulo.oa?id=72157132006

Herrera, T. (2014). De las limpiezas de ríos a la conservación y mantenimiento de ríos: prevención de riesgos, conservación y empleo pueden darse la mano. In Boletín Especial Día Mundial del Agua. CIREF, 24 marzo 2014. Retrieved from https://fnca.eu/images/documentos/DOCUMENTOS/Tony_Herrera.pdf

Hooke, R. L., Martín-Duque, J. F., \& Pedraza, J. (2012). Land transformation by humans: a review. GSA today, 22(12), 4-10.

Hussein, H. (2006). Urban recreational riverfronts: Successful revitalisation elements. Journal of Design and Built Environment, 2(1). 
Instituto Geológico y Minero de España. Consorcio de compensación de seguros (2004). Análisis del impacto de los riesgos geológicos en España. Evaluación de pérdidas por terremotos e inundaciones en el periodo 1987-2001 y estimación para el periodo 2004-2033. Madrid.

Intergovernmental Panel on Climate Change (IPCC). Climate Change 2013: The Physical Science Basis. Contribution of Working Group I to the Fifth Assessment Report of the Intergovernmental Panel on Climate Change. Cambridg: Cambridge University Press.

Kellner, E., \& Hubbart, J. A. (2016). A comparison of the spatial distribution of vadose zone water in forested and agricultural floodplains a century after harvest. Science of the Total Environment, 542, 153-161.

Kline, M., \& Cahoon, B. (2010). Protecting River Corridors in Vermont 1. JAWRA Journal of the American Water Resources Association, 46(2), 227-236.

Knoll, M., Lubken, U., \& Schott, D. (Eds.) (2017). Rivers Lost, Rivers Regained: Rethinking CityRiver Relations. University of Pittsburgh Press.

Kondolf, G. M., Podolak, K., \& Grantham, T. E. (2013). Restoring mediterranean-climate rivers. Hydrobiologia, 719(1), 527-545.

Kondolf, G. M., \& Pinto, P. J. (2017). The social connectivity of urban rivers. Geomorphology, 277, 182-196.

Kutschker, A., Brand, C., \& Miserendino, M. L. (2009). Evaluación de la calidad de los bosques de ribera en ríos del NO del Chubut sometidos a distintos usos de la tierra. Ecología austral, 19(1), 19-34.

Lerner, D. N., \& Holt, A. (2012). How should we manage urban river corridors? Procedia Environmental Sciences, 13, 721-729.

Lewin, J. (2013). Enlightenment and the GM floodplain. Earth Surface Processes and Landforms, 38(1), 17-29.

Long, H., Tang, G., Li, X., \& Heilig, G. K. (2007). Socio-economic driving forces of land-use change in Kunshan, the Yangtze River Delta economic area of China. Journal of Environmental management, 83(3), 351-364.

López-García. J., Camarasa, B., \& Mateu, J. F. (2007). Cambios en los usos del suelo y producción de escorrentía en ramblas mediterráneas: Carraixet y Poyo (1956-1998). Boletín de la 
Asociación de Geógrafos Españoles, 44, 69-94. Retrieved from https://bage.agegeografia.es/ojs/index.php/bage/article/view/610

Madsen, H., Lawrence, D., Lang, M., Martinkova, M., \& Kjeldsen, T. R. (2014). Review of trend analysis and climate change projections of extreme precipitation and floods in Europe. Journal of Hydrology, 519, 3634-3650.

Malavoi, J. R., \& Souchon, Y. (1989). Méthodologie de description, quantification des variables morphodynamiques d'un cours d'eau à fond caillouteux: exemple d'une station sur la Fillière (Haute-Savoie)/A methodology for the description and the quantification of the morphodynamic variables along a gravel bed river: a reach of the Fillière river (Haute-Savoie) as an example. Géocarrefour, 64(4), 252-259.

Martínez, C., Pertusa, I., Díaz, L., Carpio-Fernández, J., Magdaleno, F., Fernández, J.A., Tomé, F., \& Sánchez, F.J. (2017). El síndrome de la cuenca urbanizada: qué es y cómo tratarlo. In $7^{\circ}$ Congreso Forestal Español. Plasencia, Cáceres, Extremadura.

Mateu, J. F. (1989). Ríos y ramblas mediterráneos. Avenidas fluviales e inundaciones en la cuenca del mediterráneo. Alicante: Instituto Universitario de Geografía.

Mateu, J. F. (1990). Avenidas y riesgos de inundación en los sistemas fluviales mediterráneos de la Península Ibérica. Boletín de la Asociación de Geógrafos Españoles, (10), 45-86. Retrieved from https://dialnet.unirioja.es/servlet/articulo?codigo $=1318204$

Mateu, J. F., \& Camarasa, A. M. (2000). Las inundaciones en España en los últimos veinte años. Una perspectiva geográfica. Serie Geográfica. Universidad de Alcalá de Henares, 9, 11-15.

Menéndez Rexach, A. (2015). Delimitación de zonas inundables y planes de gestión del riesgo de inundación. Ambienta, 110, 36-43.

Mielgo, R. (2007). Urban Morphological Zones. Version F1vO. Definition and procedural steps. Copenhague, Dinamarca: Agencia Europea del Medio Amiente.

Middleton, N. (2012). Rivers: a very short introduction. OUP Oxford.

Ministerio para la Transición Ecológica. (2019). Recomendaciones para la construcción y rehabilitación de edificaciones en zonas inundables. Retrieved from https://www.miteco.gob.es/es/agua/temas/gestion-de-los-riesgos-de-inundacion/guiarecomendaciones-construccion-y-rehabilitacion-edificaciones-zonas-inundables_tcm30503724.pdf 
Munich Re Group (2006). Annual review: Natural catastrophes 2005. Knowledge series: Topics geo. Retrieved from https://www.preventionweb.net/files/1609_topics2005.pdf

Noguera, I., Duarte, P., \& Ollero, A. (2018). Caracterización hidrogeomorfológica y evaluación del riesgo en un curso efímero mediterráneo: el río Seco (Aragón, España). INDES Revista de Investigación para el Desarrollo Sustentable, 2(2), 9-19.

Nunes, F., Da Graça, M., Nunes, F., \& Ramos, I. (1999). Floodplain management in urban developing areas. Part I. Urban growth scenarios and land-use controls. Water resources management, 13(1), 1-21.

Nuñez, A., Salgado, A., Martín, D., Prieto, I., Montoya, J., Ferreiro, P., Feli, M., \& Soler, V. (2019). Recomendaciones para la construcción y rehabilitación de edificaciones en zonas inundables. Coordinated by Francisco Javier Sánchez \& Mónica Aparicio. Madrid: Ministerio para la Transición Ecológica.

Observatorio de la Sostenibilidad (2019). Población en riesgo de inundación en España en la franja de los primeros 10 kilómetros de costa. Observatorio de la Sostenibilidad en España.

Olazabal, E., \& Bellet, C. (2017). Análisis de las nuevas dinámicas de urbanización en España. Su estudio a través del uso de Corine Land Cover y SIOSE. In Actas del XXV Congreso de la Asociación de Geógrafos Españoles (pp. 2639-2648). Retrieved from https://dialnet.unirioja.es/servlet/articulo?codigo $=7366849$

Olcina, A. G. (1988). Precipitaciones y regímenes fluviales en la vertiente mediterránea española. Boletín de la Asociación de Geógrafos Españoles, (7), 1-12. Retrieved from https://dialnet.unirioja.es/servlet/articulo?codigo $=1317455$

Olcina, J. (2004). Riesgo de inundaciones y ordenación del territorio en la escala local: el papel del planeamiento urbano municipal. Boletín de la Asociación de Geógrafos Españoles, (37), 49-

84. Retrieved from https://bage.age-geografia.es/ojs/index.php/bage/article/view/1977

Olcina, J., \& Díez-Herrero, A. (2017). Cartografía de inundaciones en España. Flood maps in Spain. Estudios Geográficos, 282, 283-315. Retrieved from https://doi.org/10.3989/estgeogr.201710

Ollero, A., Ibisate, A., \& Elso, J. (2009). El territorio fluvial y sus dificultades de aplicación. Geographicalia, 56, 37-62.

Ollero A., \& Briz J.L. (2018). Ciudad y territorio fluvial en Zaragoza. In X aniversario de la Exposición Internacional. Zaragoza, December 23-25. 
Olson, D. M., \& Dinerstein, E. (1998). The Global 200: a representation approach to conserving the Earth's most biologically valuable ecoregions. Conservation biology, 12(3), 502-515.

Parveen, S., Basheer, J., \& Praveen, B. (2018). A literature review on land cover changes. International Journal of Advanced Research 6(7), 1-6. https://doi.org/10.21474/IJAR01/7327 Pellicer, F. (2005). Ordenación paisajística de espacios fluviales en las ciudades mediterráneas. In Paisaje y ordenación del territorio (pp. 283-295). Consejería de Obras Públicas y Transportes.

Piégay, H., Darby, S. E., Mosselman, E., \& Surian, N. (2005). A review of techniques available for delimiting the erodible river corridor: a sustainable approach to managing bank erosion. River research and applications, 21(7), 773-789.

Radcliffe, D. (1977). A nature conservation review: the selection of biological sites of national importance to nature conservation in Britain. Cambridge University Press.

Romero-Aloy, M. J. (2019). Riesgo hídrico y planeamiento urbanístico: una confusa complementariedad. ACE: Architecture, City and Environment, 40, 6588. https://doi.org/10.5821/ace. 14.40.6471

Sala, M., \& Inbar, M. (1992). Some hydrologic effects of urbanization in Catalan rivers. Catena, $19,363-378$.

Sánchez Martínez, F. J. \& Lastra, J. (Coords.) (2011). Guía metodológica para el desarrollo del Sistema Nacional de Cartografía de Zonas Inundables. Madrid: Ministerio de Medio Ambiente y Medio Rural y Marino.

Santasusagna, A., \& Tort, J. (2018). ¿Ríos urbanos, corredores verdes? Una reflexión crítica sobre la regeneración de los espacios fluviales desde una óptica ambiental. In X Congresso Ibérico de Gestão e Planeamento da Água. Coimbra.

Santos, P.M., \& Peña-Corvillon, D. (2014). Urban black holes: the rural in the urban as liminal spaces from where to build a new city. In V. Oliveira, P. Pinho, L. Batista, T. Patatas, \& C. Monteiro (Eds.), Our Common Future in Urban Morphology (pp. 995-1000). Porto: FEUP.

Tockner, K., \& Stanford, J. A. (2002). Riverine flood plains: present state and future trends. Environmental Conservation, 29, 308-330.

Thornes, J. B., \& Wainwright, J. (2004). Environmental issues in the Mediterranean: processes and perspectives from the past and present (Vol. 1). London: Routledge. 
Ureña, J. M. (2002). La ordenación de los espacios fluviales en las ciudades. In P. de la Cal Nicolás \& F. Pellicer Corellano (Coords.), Ríos y ciudades: aportaciones para la recuperación de los ríos y riberas de Zaragoza (pp. 45-63). Retrieved from hitps://ifc.dpz.es/recursos/publicaciones/22/86/05urena.pdf

Vázquez-Rodríguez, A. L. (2017). Inundaciones en la costa Atlántica y en la costa Mediterránea, dos entornos diferentes, un problema en común: estudio comparativo. Geotemas (Madrid), (17), 215-218.

Vietz, G. J., Sammonds, M. J., Walsh, C. J., Fletcher, T. D., Rutherfurd, I. D., \& Stewardson, M. J. (2014). Ecologically relevant geomorphic attributes of streams are impaired by even low levels of watershed effective imperviousness. Geomorphology, 206, 67-78.

Yagüe, J. (2010). El Sistema Nacional de Cartografía de Zonas Inundables. In Jornada sobre la nueva Directiva Europea de Inundaciones. Colegio de Caminos, Canales y Puertos, Madrid. Retrieved from http://docplayer.es/90407695-El-sistema-nacional-de-cartografia-de-zonasinundables-jesus-yague-cordova-subdirector-general-de-gestion-integrada-del-dominio-publicohidraulico.html

Yeh, A. G. O., \& Li, X. (1999). Economic development and agricultural land loss in the Pearl River Delta, China. Habitat international, 23(3), 373-390. 